\title{
Super-Exponential Blind Adaptive Beamforming
}

\author{
Kehu Yang, Member, IEEE, Takashi Ohira, Fellow, IEEE, Yimin Zhang, Senior Member, IEEE, and \\ Chong-Yung Chi, Senior Member, IEEE
}

\begin{abstract}
The objective of the beamforming with the exploitation of a sensor array is to enhance the signals of the sources from desired directions, suppress the noises and the interfering signals from other directions, and/or simultaneously provide the localization of the associated sources. In this paper, we present a higher order cumulant-based beamforming algorithm, namely, the super-exponential blind adaptive beamforming algorithm, which is extended from the super-exponential algorithm (SEA) and the inverse filter criteria (IFC). While both SEA and IFC assume noise-free conditions, this requirement is no longer needed, and all the noise components are taken into account in the proposed algorithm. Two special conditions are derived under which the proposed blind beamforming algorithm achieves the performance of the corresponding optimal nonblind beamformer in the sense of minimum mean square error (MMSE). Simulation results show that the proposed algorithm is effective and robust to diverse initial weight vectors; its performance with the use of the fourth-order cumulants is close to that of the nonblind optimal MMSE beamformer.
\end{abstract}

Index Terms-Adaptive array processing, blind beamforming, high-order cumulants, inverse filter criteria (IFC), super-exponential algorithm (SEA).

\section{INTRODUCTION}

$\mathbf{T}$ HE concept of the adaptive antenna [1] was first proposed more than three decades ago. Since then, the adaptive array or, in general, adaptive beamforming techniques, have been widely investigated for various purposes [2], [3], [20], [26]. In a variety of applications, the objective of the beamforming is to enhance the signals of the sources from desired directions, suppress the noises and the interfering signals from other directions, and/or simultaneously provide the localization of the associated source [20]. The optimal beamformer (or spatial filter), in the sense of minimum mean square error (MMSE) criteria, is specified by the Wiener-Hopf equation [2], [3], provided that the reference signal waveform or, alternatively, the

Manuscript received October 28, 2002; revised June 24, 2003. This work was supported in part by the Telecommunications Advancement Organization (TAO) of Japan. The associate editor coordinating the review of this manuscript and approving it for publication was Dr. Fulvio Gini.

K. Yang was with ATR Adaptive Communications Research Laboratories, Kyoto 619-0288, Japan. He is currently with the National Key Laboratory of Radar Signal Processing, School of Electronic Engineering, Xidian University, Xi' an 710071, China (e-mail: yang001@ xidian.edu.cn).

T. Ohira is with ATR Adaptive Communications Research Laboratories, Kyoto 619-0288, Japan (e-mail: ohira@ atr.co.jp).

Y. Zhang is with Center for Advanced Communications, Villanova University, Villanova, PA 19085, USA (e-mail: yimin@ieee.org).

C.-Y. Chi is with Department of Electrical Engineering, National Tsing Hua University, Hsinchu, Taiwan 30013 R.O.C. (e-mail: cychi@ee.nthu.edu.tw, cychi@com.nthu.edu.tw, c.y.chi@ieee.org).

Digital Object Identifier 10.1109/TSP.2004.827194 spatial signature (spatial response of the associated antenna array) of the desired source is available. However, it is often encountered in practical situations that the duration of the reference signal is not sufficiently long or that the reference signal is not available at all. The former situations happen, for example, in many mobile communication applications where the length of pilot signals is limited to maintain a high communication capacity. The later situations arise in communication countermeasure and various passive radar and sonar applications. In such situations, adaptive algorithms based on the MMSE criterion cannot work properly. As a result, it becomes increasingly important to develop blind adaptive beamforming algorithms in the absence of a reference signal.

The constant modulus algorithm (CMA) is a well-known algorithm originally developed for blind equalization [15], [16] and has recently been used for blind beamforming [17]. Instead of using training signals, the CMA uses the constant amplitude property of the desired signals. Analyses reported in [18] and [19] have shown that, in certain cases where, for instance, the source signals are of high signal-to-noise ratio (SNR) and are not closely spaced, the steady-state and convergence performances of the CMA-based blind beamforming algorithm are very close to those obtained from the corresponding nonblind ones. However, the CMA-based blind beamformers usually incur considerable performance degradation when these conditions are not satisfied.

Applying higher order statistics, which are usually described in terms of cumulants [7], or cyclostationary properties of signals to blind beamforming is considered to be a new trend to effectively make use of the inherent properties of the signals [11]-[14]. However, the methods reported in the open literature so far are only applicable to certain specific signal environments. For example, the kurtosis (fourth-order cumulant) maximization algorithm [11] globally converges only in noise-free situations. The fourth-order cumulant-based blind beamforming approach [12], [13] assumes that the interfering signals are Gaussian distributed, that incoming signals are coherent, or that the incoming signals are independent and with the identical fourth-order cumulants. On the other hand, to apply cyclostationarity in blind adaptive beamforming, $a$ priori knowledge of the exact cyclic frequency of the desired signal is required [14]. Adaptive beamforming algorithms based on cyclic correlation do not work properly when the cyclic frequency of the desired source signal is not accurately estimated [14].

The recent development of the cumulants-based blind deconvolution algorithms, namely, the super-exponential algorithm (SEA) [4], [6] and the inverse filter criteria (IFC) [5], [8], [9], opened a new avenue for blind deconvolution and equalization 
[4]. The extensions of SEA [6] to multi-input multi-output (MIMO) blind deconvolution problems proved to be effective for blind equalization in multiuser wireless communications [10], [22]-[24]. All the formulations of these single-input single-output (SISO) or MIMO blind deconvolution problems make the following assumptions:

1) infinite length of the overall channel (convolution of the channel and the equalizer);

2) finite length of the FIR filters;

3) the absence of noise.

The noise-free assumption is basically justified by using a highinput SNR. According to [6], [10], and [22]-[24], an implicit condition required for formulating the SEA is that the number of independent sources (which also include independent intersymbol interference components, if there are any) must exceed the degrees of freedom (DOFs) of the system [3]. Violation of this condition will result in failure to apply SEA because the corresponding correlation matrix is rank deficient and, therefore, noninvertible [6], [22], [23]. This is a strict constraint and is in conflict with the well-known requirement that the system DOFs must exceed the number of independent sources for effective interference suppression. Situations with the number of the independent sources being less than either the number of array sensors or the number of the total taps of the space-time FIR filters are often encountered in various communications and radar applications. In spite of such importance, applications of SEA to such practical scenarios have not been well investigated thus far.

Another very important factor to be considered is the system noise, which highly limits the system performance. In practice, noise is always present, and the input SNR could be moderate or even low (examples of such situations include countermeasure in communications, radar, and sonar, etc.). This fact underscores the importance of developing new formulations of the SEA with the noises taken into account.

The purpose of this paper is to formulate an SEA-based blind beamforming algorithm with the consideration of array noise and interference signals. The proposed algorithm works robustly, independent of whether the number of independent sources is more or less than the DOFs of the array system. Without loss of generality, we confine our formulation to the classical adaptive beamforming for independent non-Gaussian source signals.

The main contributions of this paper are three fold. First, this paper presents a super-exponential blind beamforming algorithm with the consideration of both noise and interference signals. Second, two special conditions are derived for the algorithm to converge to the optimal beamformer specified by the Wiener-Hopf solution. Third, this paper proves that the inverse filter criteria [8], [9] can be used as the objective function for the proposed beamforming algorithm, and a complete blind beamforming algorithm is subsequently obtained.

The paper is organized as follows. In Section II, we formulate the super-exponential blind beamforming algorithm and prove the two special conditions. Section III reveals the relationship between the proposed algorithm and the existing IFC, and the complete blind beamforming algorithm is then derived. Sec- tion IV presents simulation results of the conventional applications. Section V concludes this paper.

\section{FORMULATION OF BLIND ADAPTIVE BEAMFORMING}

\section{A. Formulation of Nonblind Adaptive Beamforming}

Let us consider an antenna array of $N(N>1)$ elements with $P(P \geq 1)$ incident non-Gaussian sources. The signal vector $\mathbf{x}(t)$ at the antenna array is expressed as

$$
\mathbf{x}(t)=\sum_{p=1}^{P} \Gamma \mathbf{a}\left(\theta_{p}\right) b_{p}(t)+\vec{\varpi}(t)
$$

where $\mathbf{a}(\theta)$ and $\vec{\varpi}(t)=\left[\varpi_{1}(t), \ldots, \varpi_{N}(t)\right]^{T}$ (the superscript "T" denotes transpose) are the array steering vector and the array noise vector, respectively, and $\Gamma=\operatorname{diag}\left\{\varsigma_{i} e^{j \varphi_{i}}, i=1, \ldots, N\right\}$ is a diagonal matrix denoting array channels' complex gains (gains and phases). The steering vector, for example, of a half-wavelength spaced linear array can be expressed as

$$
\begin{aligned}
\mathbf{a}(\theta) & =\left[e^{-j(2 \pi / \lambda)((N-1) / 2) d \sin (\theta)}, e^{-j(2 \pi / \lambda)((N-3) / 2) d \sin (\theta)}\right. \\
& \left., \ldots, e^{j(2 \pi / \lambda)((N-3) / 2) d \sin (\theta)}, e^{j(2 \pi / \lambda)((N-1) / 2) d \sin (\theta)}\right]^{T}
\end{aligned}
$$

with the phase reference point taken at the center of the array. To simplify the notation without loss of generality, hereafter, let us use $\mathbf{a}(\theta)$ instead of $\Gamma \mathbf{a}(\theta)$.

The following assumptions are made for the sources' signals and the employed antenna array.

A1) The source signals $b_{p}(t), p=1, \ldots, P$ are independent, stationary, and non-Gaussian processes with zero mean and variance $E\left|b_{p}(t)\right|^{2}=\sigma_{b_{p}}^{2}$.

A2) The noise vector $\vec{\varpi}(t)$ is zero-mean, spatially white (but not limited) with $E\left\{\vec{\varpi}(t) \vec{\varpi}^{T}(t)\right\}=0$ and $E\left\{\vec{\varpi}(t) \vec{\varpi}^{H}(t)\right\}=\sigma_{\varpi}^{2} \mathbf{I}_{N \times N}$, where the superscript " $H$ " denotes conjugate transpose, $\sigma_{\varpi}^{2}$ expresses the noise power at each element, and $\mathbf{I}_{N \times N}$ is the $N \times N$ identity matrix. $\vec{\varpi}(t)$ is uncorrelated with all the source signals.

A3) The steering vectors of all the associated sources are linearly independent when $P \leq N$.

For the purpose of later use, we denote $b_{p}(t)=\sigma_{b_{p}} \bar{b}_{p}(t)$, $p=1, \ldots, P, \varpi_{l}(t)=\sigma_{\varpi} \bar{\varpi}_{l}(t), l=1, \ldots, N, \mathbf{I}_{N \times N}=$ $\left[\mathbf{e}_{N \times 1}^{(1)}, \ldots, \mathbf{e}_{N \times 1}^{(N)}\right]$, and $\overline{\mathbf{A}}(\Theta)=\left[\sigma_{b_{1}} \mathbf{a}\left(\theta_{1}\right), \ldots, \sigma_{b_{P}} \mathbf{a}\left(\theta_{P}\right)\right]$, and we define

$$
\begin{aligned}
\mathbf{H} & =\left[\sigma_{b_{1}} \mathbf{a}\left(\theta_{1}\right), \ldots, \sigma_{b_{P}} \mathbf{a}\left(\theta_{P}\right), \sigma_{\varpi} \mathbf{e}_{N \times 1}^{(1)}, \ldots, \sigma_{\varpi} \mathbf{e}_{N \times 1}^{(N)}\right] \\
& =\left[\overline{\mathbf{A}}(\Theta)_{N \times P} \sigma_{\varpi} \mathbf{I}_{N \times N}\right]_{N \times(N+P)}
\end{aligned}
$$

and

$$
\begin{aligned}
\vec{\alpha}(t) & =\left[\alpha_{1}(t), \ldots, \alpha_{P+N}(t)\right]^{T} \\
& =\left[\bar{b}_{1}(t), \ldots, \bar{b}_{P}(t), \bar{\varpi}_{1}(t), \ldots, \bar{\varpi}_{N}(t)\right]^{T} .
\end{aligned}
$$

Then

$$
E\left[\vec{\alpha}(t) \vec{\alpha}^{H}(t)\right]=\mathbf{I}_{(P+N) \times(P+N)} .
$$


With straightforward manipulations, the signal vector of (1) can be expressed as the following linear vector model:

$$
\begin{aligned}
\mathbf{x}(t)= & {\left[\sigma_{b_{1}} \mathbf{a}\left(\theta_{1}\right), \ldots, \sigma_{b_{P}} \mathbf{a}\left(\theta_{P}\right), \sigma_{\varpi} \mathbf{e}_{N \times 1}^{(1)}, \ldots, \sigma_{\varpi} \mathbf{e}_{N \times 1}^{(N)}\right] } \\
& \times\left[\begin{array}{c}
\bar{b}_{1}(t) \\
\vdots \\
\bar{b}_{P}(t) \\
\bar{\varpi}_{1}(t) \\
\vdots \\
\bar{\varpi}_{N}(t)
\end{array}\right] \\
= & \mathbf{H}_{N \times(N+P)} \vec{\alpha}(t)_{(N+P) \times 1} .
\end{aligned}
$$

In order to avoid confusion between the subsequent SEA extension and that presented in [6], [10], and [22]-[24], we highlight the following two cases for ease of understanding.

Case 1) $1 \leq P<N$. This represents the kind of typical cases in communications, radar, and sonar applications.

Case 2) $P \geq N$. This is the case where the associated system does not have enough DOFs.

According to [6], [10], and [22]-[24], an implicit condition necessary to formulate the exsiting SEA is that the number of independent sources (including independent intersymbol components, if there are any) is greater than the DOFs of the system [3]. It is obvious that Case 2 satisfies this requirement. The abovementioned extension [6], [10], [22]-[24] can be directly applied to Case 2, regardless of whether the system noises are considered or not.

Remark 1: Note that in the absence of noises and for $P \leq N$ (i.e., the assumption A2 in [23]), the signal model expressed by (1) and (5) is a special case of [23, (1) and (2)] by setting $k=0$. It is clear that $\mathbf{H}$ in (5) is of full column rank if $1 \leq P<N$ as the noises are absent.

In this paper, we concentrate on Case 1 with the presence of noise. In (5), the dimension of the combined signal vector $\vec{\alpha}$ is $(N+P) \times 1$, which, regardless of the value of $N$ and $P$, is always greater than $N$.

Using assumptions $\mathrm{A} 1$ and $\mathrm{A} 2$, the correlation matrix of the array signal vector of $\mathbf{x}(t)$ can be expressed as

$$
\begin{aligned}
\mathbf{R} & =E\left[\mathbf{x}(t) \mathbf{x}^{H}(t)\right]=\mathbf{H} E\left[\vec{\alpha}(t) \vec{\alpha}^{H}(t)\right] \mathbf{H}^{H}=\mathbf{H H}^{H} \\
& =\overline{\mathbf{A}}(\Theta) \overline{\mathbf{A}}^{H}(\Theta)+\sigma_{\varpi}^{2} \mathbf{I}_{N \times N} .
\end{aligned}
$$

The output of adaptive antenna is formulated as

$$
z(t)=\mathbf{c}^{T} \mathbf{x}(t)=\mathbf{c}^{T} \mathbf{H} \vec{\alpha}(t)
$$

where $\mathbf{c}=\left[c_{1}, c_{2}, \ldots, c_{N}\right]^{T}$ is the complex weight vector. Define

$$
\mathbf{s}=\left[\begin{array}{lll}
s_{1} & \cdots & s_{P+N}
\end{array}\right]^{T}=\mathbf{H}^{T} \mathbf{c}_{N \times 1}
$$

as the gain vector of all the signals and the noises. Then, the output of the adaptive antenna can be rewritten as

$$
z(t)=\mathbf{c}^{T} \mathbf{x}(t)=\mathbf{s}^{T} \vec{\alpha}(t)=\sum_{n=1}^{P+N} s_{n} \alpha_{n}(t) .
$$

Note that there are only $N$ weights that are actually controllable, and the gains corresponding to all the signals and the noises depend on the $N$ controllable weights.

Assume that $b_{n_{0}}(t), 1 \leq n_{0} \leq P$ is the source signal of interest. Using assumptions A1 and A2, the mean square error (MSE) between $b_{n_{0}}(t)$ and the output of the adaptive antenna can be expressed as

$$
\begin{aligned}
\text { MSE } & =E\left(\left|b_{n_{0}}(t)-z(t)\right|^{2}\right)=\left|s_{n_{0}}-\sigma_{b_{n_{0}}}\right|^{2}+\sum_{\substack{n=1 \\
n \neq n_{0}}}^{P+N}\left|s_{n}\right|^{2} \\
& =\left\|\mathbf{s}-\sigma_{b_{n_{0}}} \mathbf{e}_{(P+N) \times 1}^{\left(n_{0}\right)}\right\|^{2}=\left\|\mathbf{H}^{T} \mathbf{c}-\sigma_{b_{n_{0}}} \mathbf{e}_{(P+N) \times 1}^{\left(n_{0}\right)}\right\|^{2} .
\end{aligned}
$$

Minimizing the MSE with respect to $\mathrm{c}$ results in the optimal weight vector, represented by the following Wiener-Hopf equation, which is called the optimal adaptive beamformer or MMSE beamformer [2], [3], [26]:

$$
\mathbf{c}_{o p t}=\left(\mathbf{H H}^{H}\right)^{-1}\left(\sigma_{b_{n_{0}}} \mathbf{H}^{*} \mathbf{e}_{(P+N) \times 1}^{\left(n_{0}\right)}\right)=\left(\mathbf{R}^{T}\right)^{-1} \mathbf{d}
$$

where

$$
\mathbf{d}=\sigma_{b_{n_{0}}} \mathbf{H}^{*} \mathbf{e}_{(P+N) \times 1}^{\left(n_{0}\right)}=\sigma_{b_{n_{0}}}^{2} \mathbf{a}^{*}\left(\theta_{n_{0}}\right)
$$

where the superscript “*” denotes complex conjugate.

\section{B. Super-Exponential Iteration Principle}

Without loss of generality, we present the super-exponential iteration principle in the same manner as that of [6] with the use of a finite dimensional complex gain vector $\mathbf{s}$. Define

$$
\begin{aligned}
& s_{n}^{\prime}=s_{n}^{p}\left(s_{n}^{*}\right)^{q}, \quad n=1, \ldots, P+N \\
& s_{n}^{\prime \prime}=\frac{1}{\left\|\mathbf{s}^{\prime}\right\|} s_{n}^{\prime}, \quad n=1, \ldots, P+N
\end{aligned}
$$

where $\mathbf{s}^{\prime}=\left[s_{1}^{\prime}, \ldots, s_{P+N}^{\prime}\right]^{T}$, and $\left\|\mathbf{s}^{\prime}\right\|=\sqrt{\sum_{n=1}^{P+N}\left|s_{n}^{\prime}\right|^{2}}$ is the norm of $\mathbf{s}^{\prime}$. Substituting $s_{n}=s_{n}^{(k-1)}$ and $s_{k}=s_{n}$ into (13a) and (13b), we obtain the following iteration equation:

$$
s_{n}^{(k)}=\frac{\left(s_{n}^{(k-1)}\right)^{p}\left(s_{n}^{(k-1) *}\right)^{q}}{\sqrt{\sum_{l=1}^{P+N}\left|\left(s_{l}^{(k-1)}\right)^{p}\left(s_{l}^{(k-1) *}\right)^{q}\right|^{2}}}, n=1, \ldots, P+N .
$$

In the case that $s_{n}^{(0)}=s_{n}=\left|a_{n}\right| e^{j \varphi_{n}}, a_{n} \neq 0, n=1, \ldots, P+$ $N$, for $k \geq 1$, we have

$$
s_{n}^{(k)}=\frac{\left|a_{n}\right|^{(p+q)^{k}} e^{j(p-q)^{k} \varphi_{n}}}{\sqrt{\sum_{l=1}^{P+N}\left|a_{l}\right|^{2(p+q)^{k}}}}, n=1, \ldots, P+N .
$$

When $p+q \geq 2$ and $p-q=1$, if $\left|a_{n_{0}}\right|>\left|a_{n}\right|, \forall n \neq n_{0}$, then, as $k \rightarrow \infty, s_{n}^{(k)}$ goes to

$$
\left\{\begin{array}{l}
s_{n_{0}}^{(k)} \stackrel{k \rightarrow \infty}{\rightarrow} e^{j \varphi_{n_{0}}} \\
\left|s_{n}^{(k)}\right| \stackrel{k \rightarrow \infty}{\rightarrow} 0, \quad n=1, \ldots, P+N, n \neq n_{0}
\end{array} .\right.
$$


From (15), it is seen that $s_{n}$ is forced to super-exponentially converge to the desired response, i.e., the magnitude of the leading component approaches 1 , and the others approach 0 . For more general cases, let us reconsider the iteration procedure with a finite-modulus complex weighting sequence, and then, (13a) and (13b) become

$$
\begin{aligned}
& s_{n}^{\prime}=\beta_{n} s_{n}^{p}\left(s_{n}^{*}\right)^{q}, \quad n=1, \ldots, P+N \\
& s_{n}^{\prime \prime}=\frac{1}{\left\|\mathbf{s}^{\prime}\right\|} s_{n}^{\prime}, \quad n=1, \ldots, P+N
\end{aligned}
$$

where $\beta_{n}=\left|\beta_{n}\right| e^{j \psi_{n}},\left|\beta_{n}\right|<\infty$, and $n=1, \ldots, P+N$. For $k \geq 1$, the equation corresponding to (14) becomes

$$
s_{n}^{(k)}=\frac{\beta_{n}\left(s_{n}^{(k-1)}\right)^{p}\left(s_{n}^{(k-1) *}\right)^{q}}{\sqrt{\sum_{l=1}^{P+N}\left|\beta_{l}\left(s_{l}^{(k-1)}\right)^{p}\left(s_{l}^{(k-1) *}\right)^{q}\right|^{2}}}, n=1, \ldots, P+N .
$$

Similar to (15), in the case where $s_{n}^{(0)}=s_{n}=\left|a_{n}\right| e^{j \varphi_{n}}, n=$ $1, \ldots, P+N$, for $k \geq 1$, we have

$$
\begin{aligned}
& s_{n}^{(k)}=\frac{\left|\beta_{n}\right|^{\sum_{l=0}^{k-1}(p+q)^{l}}\left|a_{n}\right|^{(p+q)^{k}} e^{j(p-q)^{k} \varphi_{n}+j \psi_{n} \sum_{l=0}^{k-1}(p-q)^{l}}}{\sqrt{\sum_{l=1}^{P+N}\left|\beta_{l}\right|^{2 \sum_{l=0}^{k-1}(p+q)^{l}}\left|a_{l}\right|^{2(p+q)^{k}}}} \\
& n=1, \ldots, P+N \text {. }
\end{aligned}
$$

When $p+q \geq 2$, (19) can be rewritten as

$$
\begin{array}{r}
s_{n}^{(k)}=\frac{\left|\beta_{n}\right|^{\left((p+q)^{k}-1\right) /(p+q-1)}\left|a_{n}\right|^{(p+q)^{k}} e^{j(p-q)^{k} \varphi_{n}+j \psi_{n} \sum_{l=0}^{k-1}(p-q)^{l}}}{\sqrt{\sum_{l=1}^{P+N}\left|\beta_{l}\right|^{2 \times\left((p+q)^{k}-1\right) /(p+q-1)}\left|a_{l}\right|^{2(p+q)^{k}}}} \\
n=1, \ldots, P+N . \quad \text { (20) }
\end{array}
$$

The convergent behavior of the above iteration sequence is described by the following theorem.

Theorem 1: For an arbitrary weighting sequence with elements of finite modulus, i.e., $\beta_{n}=\left|\beta_{n}\right| e^{j \psi_{n}},\left|\beta_{n}\right|<\infty$, $n=1, \ldots, P+N$, the conditions for the iterative updated vector (20) to super-exponentially converge to a vector that the magnitude of the leading component equal to 1 and the other components equal to 0 , i.e.,

$$
\left\{\begin{array}{l}
\left|s_{n_{0}}^{(k)}\right| \stackrel{k \rightarrow \infty}{\rightarrow} 1 \\
s_{n}^{(k)} \mid \stackrel{k \rightarrow \infty}{\rightarrow} 0, \quad n=1, \ldots, P+N, n \neq n_{0}
\end{array}\right.
$$

are $p+q \geq 2$, and

$$
\begin{aligned}
\left|a_{n_{0}}\right|\left|\beta_{n_{0}}\right|^{1 /(p+q-1)}>\left|a_{n}\right|\left|\beta_{n}\right|^{1 /(p+q-1)} & \\
n & =1, \ldots, P+N, n \neq n_{0} .
\end{aligned}
$$

The proof of Theorem 1 is given in Appendix A.

\section{Formulation of Blind Adaptive Beamforming}

It is seen that for $k \geq 1$, the associated gain vector is updated by (8) with a given weight vector $\mathbf{c}^{(k)}$, i.e.,

$$
\mathbf{s}^{(k)}=\left[\begin{array}{lll}
s_{1}^{(k)} & \ldots & s_{P+N}^{(k)}
\end{array}\right]^{T}=\mathbf{H}^{T} \mathbf{c}^{(k)} .
$$

On the other hand, the gain $s_{n}^{(k) \prime}$ can be directly updated based on $s_{n}^{(k-1)}$, i.e., $s_{n}^{(k) \prime}=\beta_{n}\left(s_{n}^{(k-1)}\right)^{p}\left(s_{n}^{(k-1) *}\right)^{q}$, $n=1, \ldots, P+N$ in accordance with (17a) without $\mathbf{c}^{(k)}$. This implies that there are evident differences between $s_{n}^{(k)}$ of (23) and $s_{n}^{(k) \prime}$ of (17a). However, due to the attractive property of the super-exponential convergence shown in Theorem 1, it is desired that the gains $s_{n}^{(k)}, n=1, \ldots, P+N$ be updated under (17a) and (17b). The best way to solve the problem is to combine the iteration procedures of (17a) and (17b) with that of (23) and then to minimize all the differences. This leads to the following constrained minimum least square (LS) problem, i.e.,

$$
\begin{aligned}
\min _{\mathbf{c}^{(k)}} \sum_{n=1}^{P+N}\left|s_{n}^{(k)}-\beta_{n}\left(s_{n}^{(k-1)}\right)^{p}\left(s_{n}^{(k-1) *}\right)^{q}\right|^{2} \\
\text { subject to } \sum_{n=1}^{P+N}\left|s_{n}^{(k)}\right|^{2}=1 .
\end{aligned}
$$

Define

$$
\begin{aligned}
\mathbf{g}^{(k-1)}=\left[\beta_{1}\left(s_{1}^{(k-1)}\right)^{p}\left(s_{1}^{(k-1) *}\right)^{q}\right. \\
\left.\ldots, \beta_{P+N}\left(s_{P+N}^{(k-1)}\right)^{p}\left(s_{P+N}^{(k-1) *}\right)^{q}\right]^{T} .
\end{aligned}
$$

Then, substituting (23) into (24) yields

$$
\min _{\mathbf{c}^{(k)}}\left\|\mathbf{H}^{T} \mathbf{c}^{(k)}-\mathbf{g}^{(k-1)}\right\|^{2}, \quad \text { subject to }\left\|\mathbf{H}^{T} \mathbf{c}^{(k)}\right\|=1 .
$$

With the use of the method of Lagrange multipliers, the solution for $\mathbf{c}^{(k)}$ can be derived straightforwardly as

$$
\mathbf{c}^{(k)}=\frac{\left(\mathbf{H}^{*} \mathbf{H}^{T}\right)^{-1} \mathbf{H}^{*} \mathbf{g}^{(k-1)}}{\sqrt{\left(\mathbf{H}^{*} \mathbf{g}^{(k-1)}\right)^{H}\left(\mathbf{H}^{*} \mathbf{H}^{T}\right)^{-1} \mathbf{H}^{*} \mathbf{g}^{(k-1)}}} .
$$

Define

$$
\mathbf{d}^{(k-1)}=\mathbf{H}^{*} \mathbf{g}^{(k-1)}
$$

and with the definitions of (6) and (27), (28) can also be expressed as

$$
\mathbf{c}^{(k)}=\frac{\left(\mathbf{R}^{T}\right)^{-1} \mathbf{d}^{(k-1)}}{\sqrt{\left(\mathbf{d}^{(k-1)}\right)^{H}\left(\mathbf{R}^{T}\right)^{-1} \mathbf{d}^{(k-1)}}}
$$

where $\mathbf{R}$ can be estimated from the samples (or array snapshots) of $\mathbf{x}(t)$, and $\mathbf{d}^{(k-1)}$ will be estimated subsequently through a high-order cumulant. Regarding (26), (27), and (29), we have the following remark.

Remark 2: As mentioned in Remark 1, in the absence of noise, $\mathbf{H}$ is of full column rank when $1 \leq P<N$. In this case, the correlation matrix $\mathbf{R}$ is rank-deficient. This implies that in such a case, it is difficult to obtain an analytical solution for the blind beamformer $\mathbf{c}^{(k)}$ from (26). As a result, the iteration procedure given by $[23,(39)$ and (40)] becomes inapplicable. 
Therefore, it is necessary to take the noises into account, and treating the noise components as independent sources is a convenient and effective way to solve the problem for Case 1 .

Next, we present the method of estimating $\mathbf{d}^{(k-1)}$. First of all, let cum $\left(x_{1} ; x_{2} ; \cdots ; x_{L}\right)$ denote the joint cumulant of $L$ random variables $\left\{x_{1}, x_{2}, \ldots, x_{L}\right\}$, and define

$$
\begin{aligned}
& \operatorname{cum}\left(z^{(l)}(t): p ; z^{(l) *}(t): q ; \mathbf{x}^{*}(t)\right) \\
& =\operatorname{cum}(\underbrace{z^{(l)}(t) ; \cdots ; z^{(l)}(t)}_{p \text { terms }} ; \underbrace{z^{(l) *}(t) ; \cdots z^{(l) *}(t)}_{q \text { terms }} ; \mathbf{x}^{*}(t)
\end{aligned}
$$

where $z^{(k)}(t)$ is the output of the beamformer $\mathbf{c}^{(k)}$ at the $k$ th iteration, i.e.,

$$
z^{(k)}(t)=\mathbf{c}^{(k) T} \mathbf{x}(t)=\mathbf{s}^{(k) T} \vec{\alpha}(t)=\sum_{n=1}^{P+N} s_{n}^{(k)} \alpha_{n}(t) .
$$

Using the linearity property of cumulants [7], i.e., $\operatorname{cum}\left(\sum_{i} b_{i} x_{i} ; \cdots\right)=\sum_{i} b_{i} \operatorname{cum}\left(x_{i} ; \cdots\right), \quad$ and substituting (31) and (5) into (30), (30) can be rewritten as (32), shown at the bottom of the page. Define

$$
\beta_{n}=\operatorname{cum}\left(\alpha_{n}(t): p ; \alpha_{n}^{*}(t): q+1\right), \mathrm{n}=1, \ldots, P+N
$$

as the cumulants of order $(p, q+1)$ of all sources. From (25), (28), and (32), we conclude that

$$
\mathbf{d}^{(k-1)}=\operatorname{cum}\left(z^{(k-1)}(t): p ; z^{(k-1) *}(t): q ; \mathbf{x}^{*}(t)\right) .
$$

According to (31), $\mathbf{d}^{(k-1)}$ can be estimated by $\mathbf{c}^{(k-1)}$ and the data samples $\mathbf{x}(t)$.

Equations (29), (31), and (34), rewritten as

$$
\begin{aligned}
\mathbf{c}^{(k)} & =\frac{\left(\mathbf{R}^{T}\right)^{-1} \mathbf{d}^{(k-1)}}{\sqrt{\left(\mathbf{d}^{(k-1)}\right)^{H}\left(\mathbf{R}^{T}\right)^{-1} \mathbf{d}^{(k-1)}}} \\
\mathbf{d}^{(k-1)} & =\operatorname{cum}\left(z^{(k-1)}(t): p ; z^{(k-1) *}(t): q ; \mathbf{x}^{*}(t)\right) \\
z^{(k-1)}(t) & =\left(\mathbf{c}^{(k-1)}\right)^{T} \mathbf{x}(t)
\end{aligned}
$$

define the super-exponential blind beamfoming algorithm. A remark on it is given as follows.
Remark 3: It is noted that the above blind super-exponential beamfoming algorithm is valid for $P \geq 1$ and can be applied regardless of the array configurations and the number of nonGaussian (i.e., nonzero cumulants) sources, which, therefore, is very important to diverse applications, e.g., communications, radar, sonar, etc.

When the noises are Gaussian distributed, then for $p+q \geq 2$ and $\mathrm{n}=P+1, \ldots, P+N, \beta_{n}$ equals zero [7]. In this case, the beamforming weight vector given by (35a) can be written as

$$
\mathbf{c}^{(k)}=\frac{\sum_{i=1}^{P} \sigma_{b_{i}}\left(\mathbf{R}^{T}\right)^{-1} \mathbf{a}^{*}\left(\theta_{i}\right) \beta_{i}\left(s_{i}^{(k-1)}\right)^{p}\left(s_{i}^{(k-1) *}\right)^{q}}{\sqrt{\left(\mathbf{d}^{(k-1)}\right)^{H}\left(\mathbf{R}^{T}\right)^{-1} \mathbf{d}^{(k-1)}}} .
$$

It is evident from (36) that the iterative weight vector is a linear combination of the optimal weight vectors corresponding to all the source signals. Furthermore, substituting (36) into (8) yields

$$
\mathbf{s}^{(k)}=\frac{\sum_{i=1}^{P} \vec{\xi}\left(\theta_{i}\right) \beta_{i}\left(s_{i}^{(k-1)}\right)^{p}\left(s_{i}^{(k-1) *}\right)^{q}}{\left\|\sum_{i=1}^{P} \vec{\xi}\left(\theta_{i}\right) \beta_{i}\left(s_{i}^{(k-1)}\right)^{p}\left(s_{i}^{(k-1) *}\right)^{q}\right\|}
$$

where

$$
\vec{\xi}\left(\theta_{i}\right)=\sigma_{b_{i}} \mathbf{H}^{T}\left(\mathbf{R}^{T}\right)^{-1} \mathbf{a}^{*}\left(\theta_{i}\right)
$$

is the gain vector of the optimal adaptive antenna with respect to the $i$ th source, and

$$
\left\|\vec{\xi}\left(\theta_{i}\right)\right\|^{2}=\sigma_{b_{i}}^{2} \mathbf{a}^{T}\left(\theta_{i}\right)\left(\mathbf{R}^{T}\right)^{-1} \mathbf{a}^{*}\left(\theta_{i}\right) .
$$

It is obvious that the optimum $\mathbf{s}^{(k)}$ is also a linear combination of $\vec{\xi}\left(\theta_{i}\right)$.

From (36) and (37), it is seen that the convergent beamformer $\mathbf{c}^{(k)}$ depends on the initial vector $\mathbf{c}^{(0)}, p+q$, input SNRs, the spatial responses of the sources, and spatial correlation between the sources.

We know that due to the constraint of (24), $\left|s_{n}^{(k)}\right|<1$ always hold for $k \geq 1$ and $n=1, \ldots, P+N$. If the initial weight vector $\mathbf{c}^{(0)}$ is chosen such that

$$
\left|s_{n_{0}}^{(0)}\right|>\max \left\{\left|s_{n}^{(0)}\right|, n=1, \ldots, P+N, n \neq n_{0}\right\}, 1 \leq n_{0} \leq P
$$

$$
\begin{aligned}
& \operatorname{cum}\left(z^{(k)}(t): p, z^{(k) *}(t): q, \mathbf{x}^{*}(t)\right)=\operatorname{cum}\left(z^{(k)}(t): p ; z^{(k) *}(t): q ; \mathbf{H}^{*} \vec{\alpha}^{*}(t)\right) \\
& =\mathbf{H}^{*} \operatorname{cum}\left(z^{(k)}(t): p ; z^{(k) *}(t): q ; \vec{\alpha}^{*}(t)\right) \\
& =\mathbf{H}^{*} \operatorname{cum}(\underbrace{\sum_{n=1}^{P+N} s_{n}^{(k)} \alpha_{n}(t) ; \cdots ; \sum_{n=1}^{P+N} s_{n}^{(k)} \alpha_{n}(t)}_{p \text { terms }} \underbrace{\sum_{n=1}^{P+N} s_{n}^{(k) *} \alpha_{n}^{*}(t) ; \cdots ; \sum_{i=1}^{P+N} s_{n}^{(k)} * \alpha_{n}^{*}(t)}_{q \text { terms }} ; \vec{\alpha}^{*}(t)) \\
& =\mathbf{H}^{*}\left[\begin{array}{c}
\left(s_{1}^{(k)}\right)^{p}\left(s_{1}^{(k) *}\right)^{q} \operatorname{cum}\left(\alpha_{1}(t): p ; \alpha_{1}^{*}(t): q+1\right) \\
\vdots \\
\left(s_{P+N}^{(k)}\right)^{p}\left(s_{P+N}^{(k) *}\right)^{q} \operatorname{cum}\left(\alpha_{P+N}(t): p ; \alpha_{P+N}^{*}(t): q+1\right)
\end{array}\right] .
\end{aligned}
$$


then, from (18) or (37), it is obvious that for arbitrary $\left|\beta_{n}\right|<$ $+\infty, n=1, \ldots, P$

$$
\mathbf{s}^{(1)} \stackrel{p+q \rightarrow \infty}{\rightarrow} \frac{e^{j(p-q) \arg \left(s_{n_{0}}^{(0)}\right)}}{\left\|\vec{\xi}\left(\theta_{n_{0}}\right)\right\|}
$$

where $\arg \left(s_{n_{0}}^{(0)}\right)$ denotes the phase of $s_{n_{0}}^{(0)}$. Note that (40) and (41) indicate that the super-exponential blind beamforming algorithm converges faster for a larger value of $p+q$. However, a larger value of $p+q$ usually gives rise to a larger variance of the estimated $\mathbf{d}^{(k-1)}[\operatorname{see}(35 \mathrm{~b})]$ thus leading to a larger variance of the designed beamfomer. Nevertheless, for an appropriate $p+q \geq 2$, as the gain of all the undesired signals are smaller than that of the desired signal under $\mathbf{c}^{(0)}$, the super-exponential beamforming algorithm will converge fast and closely to the optimal beamformer. In other words, if $\mathbf{c}^{(0)}$ is chosen such that

$$
\mathbf{s}^{(0)}=\frac{\vec{\xi}\left(\theta_{n_{0}}\right)}{\left\|\vec{\xi}\left(\theta_{n_{0}}\right)\right\|}
$$

then under an appropriate $p+q \geq 2$, we have

$$
\mathbf{s}^{(1)}=\frac{e^{j(p-q) \arg \left(s_{n_{0}}^{(0)}\right)} \vec{\xi}\left(\theta_{n_{0}}\right)+\vec{\varepsilon}}{\left\|e^{j(p-q) \arg \left(s_{n_{0}}^{(0)}\right)} \vec{\xi}\left(\theta_{n_{0}}\right)+\vec{\varepsilon}\right\|}
$$

where $\vec{\varepsilon}$ is an error vector with $\|\vec{\varepsilon}\|$ much smaller than $\left\|\vec{\xi}\left(\theta_{n_{0}}\right)\right\|$.

In order to give more insight into the behavior and the performance of the proposed algorithm, we present two theorems in the following for two special cases, respectively. In the first case, the SNRs of all source signals approach infinity, whereas in the second, the desired source is spatially orthogonal to the other sources.

Theorem 2: When $P \leq N, p+q \geq 2$, and the noise components are Gaussian distributed, the algorithm specified by (35a)-(35c) performs perfect suppression of all the interfering signals if $\sigma_{b_{i}}^{2} / \sigma_{\varpi}^{2} \rightarrow+\infty, i=1, \ldots, P$, and

$$
\begin{array}{r}
\left|\beta_{n_{0}}\right|^{1 /(p+q-1)}\left|s_{n_{0}}^{(0)}\right|>\max \left\{\left|\beta_{n}\right|^{1 /(p+q-1)}\left|s_{n}^{(0)}\right|\right. \\
\left.n=1, \ldots, P, n \neq n_{0}\right\}
\end{array}
$$

where $\beta_{n}$ is defined by (33).

Theorem 2 is proved in Appendix B.

Remark 4: Theorem 2 implies that the higher the SNRs of all the source signals, the closer the blind beamformer approaches the nonblind optimal beamformer (the MMSE beamformer). In addition, the blind beamformer approaches the nonblind one when the SNRs of all the sources approach infinity.

Theorem 3: When $P \leq N, p+q \geq 2$, and the noise components are Gaussian distributed, the algorithm specified by (35a)-(35c) converges to the nonblind optimal (MMSE) beamformer if the steering vector of the $n_{0}$ th $\left(1 \leq n_{0} \leq P\right)$ source is spatially orthogonal to those of all the other sources, i.e.,

$$
\mathbf{a}^{T}\left(\theta_{n}\right) \mathbf{a}^{*}\left(\theta_{n_{0}}\right)=0, n=1, \ldots, P, n \neq n_{0}
$$

and

$$
\begin{aligned}
\mu_{0}\left|\beta_{n_{0}}\right|^{1 /(p+q-1)}\left|s_{n_{0}}^{(0)}\right|>\tilde{\beta}_{r_{0}}^{1 /(p+q-1)}\left|s_{m_{0}}^{(0)}\right| & \\
& n_{0} \neq m_{0}, 1 \leq m_{0}, r_{0} \leq P
\end{aligned}
$$

where

$$
\begin{aligned}
& \beta_{n}= \\
& \begin{cases}\operatorname{cum}\left(\alpha_{n}(t): p ; \alpha_{n}^{*}(t): q+1\right), & n=1, \ldots, P \\
0, & n=P+1, \ldots, P+N\end{cases} \\
& \tilde{\beta}_{r_{0}}=\max \\
& \left\{\sum_{\substack{i=1 \\
i \neq n_{0}}}^{P} \sigma_{b_{n}} \sigma_{b_{i}} \mathbf{a}^{T}\left(\theta_{n}\right)\left(\mathbf{R}^{T}\right)^{-1} \mathbf{a}^{*}\left(\theta_{i}\right)|| \beta_{i} \mid, n=1, \ldots, P, n \neq n_{0}\right\} \\
& \left|s_{m_{0}}^{(0)}\right|=\max \left\{\left|s_{n}^{(0)}\right|, n=1, \ldots, P\right\} \\
& \mu_{0}=\frac{\frac{\left\|\mathbf{a}\left(\theta_{n_{0}}\right)\right\|^{2} \sigma_{b_{n_{0}}}^{2}}{\sigma_{\varpi}^{2}}}{1+\frac{\left\|\mathbf{a}\left(\theta_{n_{0}}\right)\right\|^{2} \sigma_{b_{n_{0}}}^{2}}{\sigma_{\varpi}^{2}}}<1 .
\end{aligned}
$$

The proof is in Appendix C.

Remark 5: Note that under (45), the algorithm converges to the conventional beamformer shown by (C8) in Appendix C. This provides some insights of the performance behavior of the proposed algorithm.

\section{OBJECTIVE FunCtion OF THE BLIND ADAPTIVE BEAMFORMING}

In many adaptive processing problems, we know that appropriate objective functions, or cost functions, are very important in developing adaptive algorithms and evaluating their performance. It has been shown in [5], [8], and [9] that the following inverse filter criteria

$$
J_{m, n}=\frac{\left|C_{m, n}\{e(k)\}\right|}{\left|C_{1,1}\{e(k)\}\right|^{(m+n) / 2}}=\frac{\left|C_{m, n}\{e(k)\}\right|}{\left|E\left\{|e(k)|^{2}\right\}\right|^{(m+n) / 2}}
$$

are suitable objective functions for blind deconvolution, where $e(k)$ represents the output of the associated blind equalizer, $m+$ $n>2$, and the cumulant of $e(k)$ is defined as

$$
\begin{aligned}
C_{m, n}\{e(k)\} & =\operatorname{cum}\left\{e(k): m, e^{*}(k): n\right\} \\
= & \operatorname{cum}\{\underbrace{e(k), \ldots, e(k)}_{m \text { terms }}, \underbrace{e^{*}(k), \ldots, e^{*}(k)}_{n \text { terms }}\} .
\end{aligned}
$$

In the absence of noises, the objective function is bounded by the maximum modulus of the cumulants of all the signal components [5], [8], [9]. In this section, our purpose is to show that the inverse filter criteria can also be an objective function for the proposed blind beamforming algorithm.

Replacing $e(k)$ in (50) with the $z(t)$ in (7)-(9), one can obtain

$$
J_{m, n}=\frac{\left|C_{m, n}\{z(t)\}\right|}{\left|C_{1,1}\{z(t)\}\right|^{(m+n) / 2}}=\frac{\left|\sum_{l=1}^{P+N} \gamma_{l}^{(m, n)} s_{l}^{m}\left(s_{l}^{*}\right)^{n}\right|}{\left.\left.\left|\sum_{l=1}^{P+N} \gamma_{l}^{(1,1)}\right| s_{l}\right|^{2}\right|^{(m+n) / 2}}
$$

where

$$
\gamma_{l}^{(m, n)}=\operatorname{cum}\{\underbrace{\alpha_{l}(t), \ldots, \alpha_{l}(t)}_{m \text { terms }}, \underbrace{\alpha_{l}^{*}(t), \ldots, \alpha_{l}^{*}(t)}_{n \text { terms }}\}
$$


represents the $(m, n)$ th-order cumulant of the $l$ th source signal. According to (4), we have

$$
\gamma_{l}^{(1,1)}=\operatorname{cum}\left\{\alpha_{l}(t), \alpha_{l}^{*}(t)\right\}=E\left\{\left|\alpha_{l}(t)\right|^{2}\right\}=1 .
$$

Defining

$$
\delta_{s}=\left.\left.\left|\sum_{l=1}^{P+N}\right| s_{l}\right|^{2}\right|^{(1 / 2)}=\left(\mathbf{c}^{T} \mathbf{R} \mathbf{c}^{*}\right)^{(1 / 2)}
$$

which is the root mean square (RMS) of the total output power, (52) can then be expressed as

$$
J_{m, n}(\mathbf{c})=\left|\sum_{l=1}^{P+N} \gamma_{l}^{(m, n)}\left(\frac{s_{l}}{\delta_{s}}\right)^{m}\left(\frac{s_{l}^{*}}{\delta_{s}}\right)^{n}\right| .
$$

Since most of the digital signal waveforms employed in communications and radar applications are of symmetric distributions, it can be shown that all their odd-order moments and cumulants are equal to zero. In the following, the situation where $m=n>1$ is considered. In this case, (56) becomes

$$
J_{2 m}(\mathbf{c})=J_{m, m}(\mathbf{c})=\left.\left|\sum_{l=1}^{P+N} \gamma_{l}^{(m, m)}\right| \frac{s_{l}}{\delta_{s}}\right|^{2 m} \mid .
$$

From the above equation, we see that for a given $n_{0}$, $1 \leq n_{0} \leq P$, the necessary and sufficient condition for $J_{2 m}(\mathbf{c})=\left|\gamma_{n_{0}}^{(m, m)}\right|$ is that $\left|s_{n_{0}}\right|=1$ and $s_{n}=0$, $n=1, \ldots, P+N, n \neq n_{0}$, which corresponds to the perfect suppression of all the interfering signals described in Theorem 2 . In practice, when the blind beamformer makes $\left|s_{n_{0}}\right|>\left|s_{n}\right|$, $n=1, \ldots, P+N, n \neq n_{0}, 1 \leq n_{0} \leq P$, then (57) can be expressed as

$$
J_{2 m}(\mathbf{c})=\left|\gamma_{n_{0}}^{(m, m)}\right|\left|\frac{s_{n_{0}}}{\delta_{s}}\right|^{2 m}\left|1+\sum_{\substack{l=1 \\ l \neq n_{0}}}^{P+N} \frac{\gamma_{l}^{(m, m)}}{\gamma_{n_{0}}^{(m, m)}}\left(\left|\frac{s_{l}}{s_{n_{0}}}\right|^{2}\right)^{m}\right|
$$

where the ratio $\left(\left|s_{l}\right|^{2} /\left|s_{n_{0}}\right|^{2}\right)^{m}$ will decrease as $m$ increases. This indicates that the larger the $m$, i.e., the higher the employed order of the cumulant, the more the reduced contributions of $\gamma_{n}^{(m)}, n=1, \ldots, P+N, n \neq n_{0}$ to $J_{2 m}(\mathbf{c})$. The term $\left|s_{n_{0}}\right|^{2} /\left|s_{l}\right|^{2}$ denotes the suppression ratio of the beamformer to the $l$ th source. The higher the suppression ratio, the smaller the contribution of the $l$ th source to the objective function.

The gradient of the objective function with respect to the weight vector is derived as

$$
\begin{aligned}
& \frac{\partial J_{2 m}(\mathbf{c})}{\partial \mathbf{c}} \\
= & \frac{\operatorname{sgn}\left(C_{m, m}\{z(t)\}\right) \times m \times \operatorname{cum}\left\{z(t): m, z^{*}(t): m-1, \mathbf{x}^{*}(t)\right\}}{\left(\mathbf{c}^{T} \mathbf{R} \mathbf{c}^{*}\right)^{m}} \\
& -\frac{m \times\left|C_{m, m}\{z(t)\}\right|}{\left(\mathbf{c}^{T} \mathbf{R} \mathbf{c}^{*}\right)^{m+1}} \times \mathbf{R}^{T} \mathbf{c}
\end{aligned}
$$

where $\operatorname{sgn}\left(C_{m, m}\{z(t)\}\right)$ expresses the sign of the cumulant $C_{m, m}\{z(t)\}$. Because $J_{2 m}(\mathbf{c})$ is a nonlinear function with respect to $\mathbf{c}$, the stationary points [5], [8], [11] of (58) can be obtained from

$$
\frac{\partial J_{2 m}(\mathbf{c})}{\partial \mathbf{c}}=\mathbf{0}
$$

We have the following theorem to show the relationship between the stationary points and the algorithm.

Theorem 4: The super-exponential iterative algorithm specified by (35a)-(35c) converges to the stationary points of the objective function (58).

Theorem 4 is proved in Appendix D.

The associated cumulant terms in (35a)-(35c) and (50) with order $(2,2),(3,3)$, and $(4,4)$ are derived in Appendix E.

From Appendix D, we see that the stationary points of the objective function cannot be expressed by an analytical solution, and the algorithm described by (35a) and (35b) satisfies (D2), which specifies the stationary points. Because the initial $\mathbf{c}^{(0)}$ could be different, the algorithm may converge to the different stationary points. In essence, Theorem 4 shows that the inverse filter criteria can be an objective function of the blind beamforming. Based on Theorem 4, the complete super-exponential blind adaptive beamforming algorithm can be cast into the following three steps.

Step 1) Let $k=1$, given the accuracy $\rho\left(=10^{-5}\right), \mathbf{c}^{(0)}$ and the order of the cumulant.

Step 2) Let $p=m$ and $q=p-1$, perform (35a)-(35c), and obtain $\mathbf{c}^{(k)}$.

Step 3) If $\left|J_{2 m}\left(\mathbf{c}^{(k)}\right)-J_{2 m}\left(\mathbf{c}^{(k-1)}\right)\right|<\rho$, then stop; Else let $k=k+1$, go to Step 1 .

\section{COMPuTER Simulations}

In order to confirm the effectiveness of the proposed algorithm, computer simulations are performed. In the simulations, a six-element uniform linear array with half-wavelength spacing is employed. The array is not calibrated, i.e., the complex gains of the array elements are perturbed from their nominal value. Herein, we assume that the perturbation gains are time-invariant, whereas their exact values are unknown. The respective nominal values of the amplitude and phase of the complex gains are set to $1(0 \mathrm{~dB})$ and $0 \mathrm{rad}$. We choose the perturbation gain from the samples of uniformly distributed random numbers, where the standard deviation of the amplitude is assumed as 0.1 , and that of the phase is assumed as $0.1 \mathrm{rad}$. As a result, the perturbation gain vector used in the simulations is randomly generated, and the set of the samples

$$
\begin{aligned}
& \{1.0236-0.1233 \mathrm{j}, 0.8636-0.0075 \mathrm{j}, 1.1470+0.1185 \mathrm{j} \\
& 1.0491-0.0626 \mathrm{j}, 0.8854-0.1041 \mathrm{j}, 1.1536+0.1937 \mathrm{j}\}
\end{aligned}
$$

is selected.

We consider the scenario where three signal sources illuminate the antenna array from the far field. All the source signals are quadrature phase shift keying signals. The strongest source is assumed to be the desired one, which is located at $12.3^{\circ}$ from the broadside of the antenna array (the broadside here is considered as the reference direction-of-arrival signals). The power of the second source is assumed to be $0.5 \mathrm{~dB}$ lower than that of the desired signal, and its incident angle is $16.5^{\circ}$ from the broadside. The power of the third source is $2 \mathrm{~dB}$ lower than that of the desired signal, and its incident angle is $-1.7^{\circ}$ from the broadside.

As mentioned in Sections II and III, different initial weight vectors may lead to different convergent results. For comparison, the following two initial weight vectors are considered. 


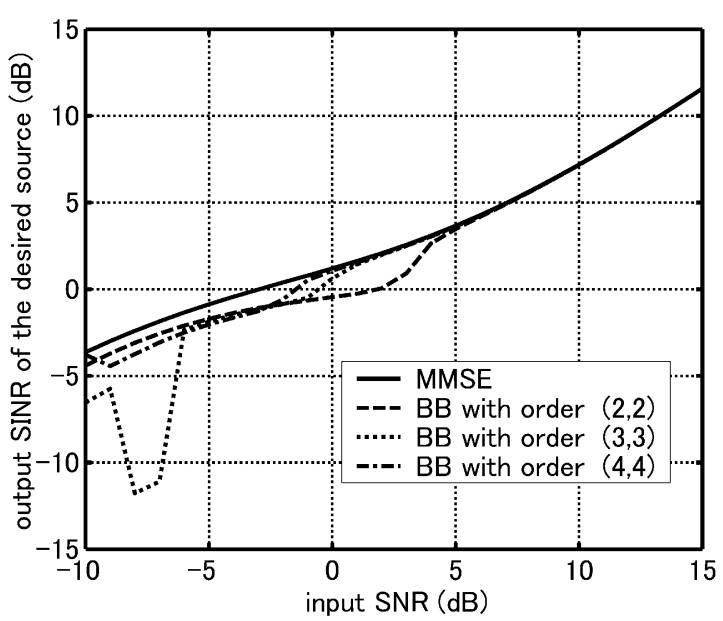

(a) under I1

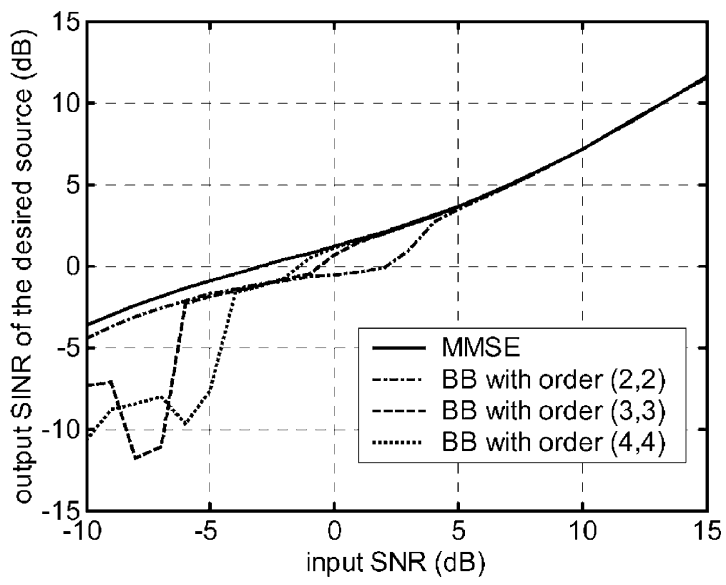

(b) under I2

Fig. 1. Output SINR of the desired source.

I1)

$$
\begin{aligned}
\mathbf{c}^{(0)}= & \left.\mathbf{a}(\theta)\right|_{\theta=12.3^{\circ}} \\
= & {\left[e^{-j(2 \pi / \lambda)(5 / 2) d \sin (\theta)}, e^{-j(2 \pi / \lambda)(3 / 2) d \sin (\theta)}\right.} \\
& \left.\ldots, e^{j(2 \pi / \lambda)(3 / 2) d \sin (\theta)}, e^{j(2 \pi / \lambda)(5 / 2) d \sin (\theta)}\right]\left.^{T}\right|_{\theta=12.3^{\circ}} .
\end{aligned}
$$

I2) $\mathbf{c}^{(0)}=[1,0, \ldots, 0]^{T}$.

It is obvious that under I1, a beam toward the desired source is formed, whereas the array response (pattern) under I2 is omnidirectional. Although better beamformer performance is expected when I1 is used, nevertheless, I 2 is often used in practice because the a priori information of the spatial signature of the desired source is usually not available.

In Fig. 1(a) and (b), we depict the output SINR versus the input SNR under the initial weight vectors I1 and I2, respectively. The number of the samples employed is 5000, and $\rho=$ $1 \times 10^{-5}$ is used. In these figures, "BB" stands for the proposed blind algorithm and "MMSE" stands for the nonblind MMSE optimal beamformer. It is evident from these figures that when the input SNR is not too low (input SNR $>-5 \mathrm{~dB}$ ), the higher the order of the cumulants used, the closer the performance of the blind algorithm approaches that of the MMSE beamformer. It is also seen that the results under I1 are slightly better than

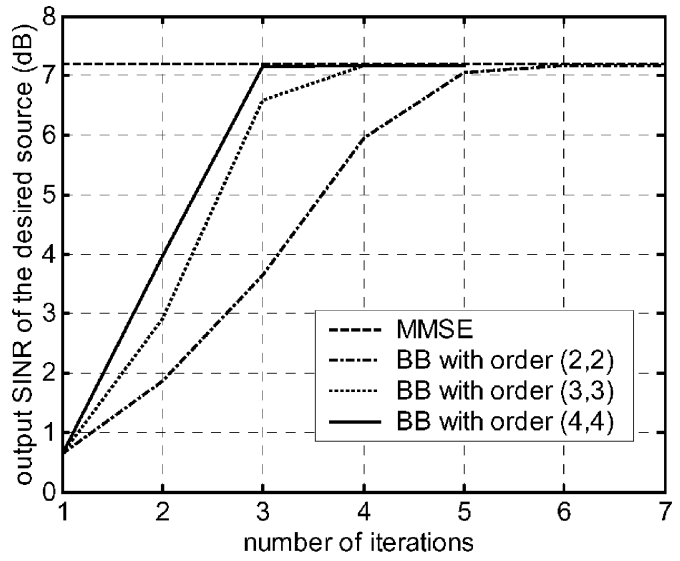

(a) under I1

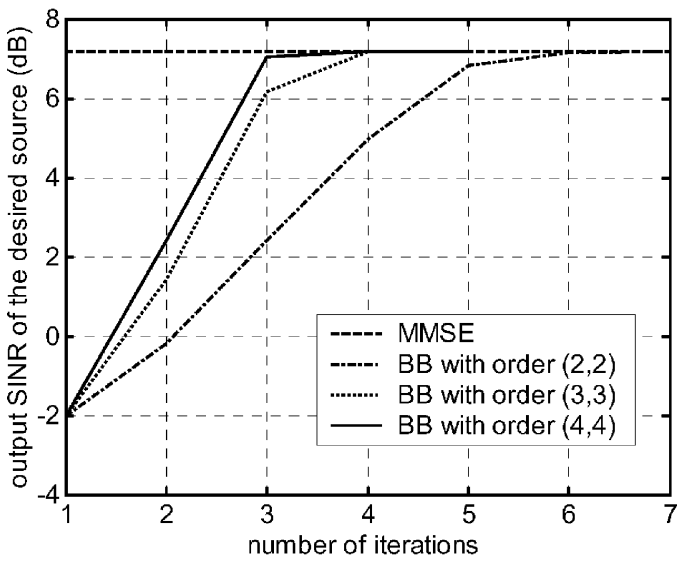

(b) under I2

Fig. 2. Comparison of the convergence performance.

that under I2. When the input SNR is high, on the other hand, all the results of the blind algorithm with both initial weight vectors I1 and I2 and different cumulant orders of (2,2), (3,3), and $(4,4)$ approach that of the MMSE beamformer with negligible difference.

Fig. 2(a) and (b) show the convergence performance of the blind algorithm under I1 and I2, respectively, where the input $\mathrm{SNR}$ is $10 \mathrm{~dB}$, and 5000 data samples are employed. From these figures, we see that the convergence rates under both the two initial weight vectors are basically the same.

To investigate the effects of the number of the array signal samples on the performance of the proposed algorithm, Fig. 3 shows the output SINR versus the number of the array data samples, where the input SNR is fixed to $10 \mathrm{~dB}$. It is seen that the longer the samples, the more closely the blind algorithm under both I1 and I 2 will converge to the optimum result. However, because the power of the second source signal is close to that of the desired source signal, it would be possible for the algorithm to mistakenly converge to the result corresponding to the second source when $\mathrm{I} 2$ is employed. Fig. 3(c) shows the phenomenon for the case of the sample size equal to 1000. This is natural because all blind algorithms are blind to the order of all the corresponding signals, provided that the a priori information is available to distinguish them.

Finally, we show the beam patterns in Fig. 4 with both initial conditions, where 5000 samples are used, and the input SNR 


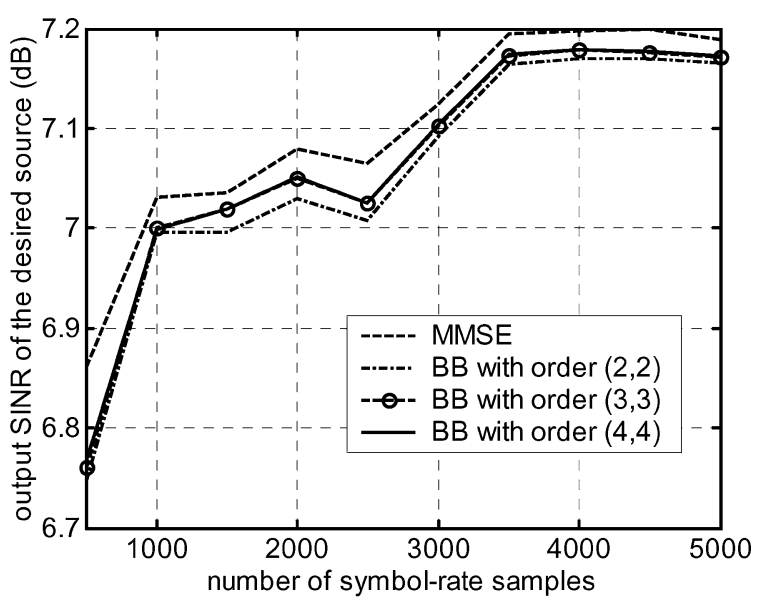

(a) under I1

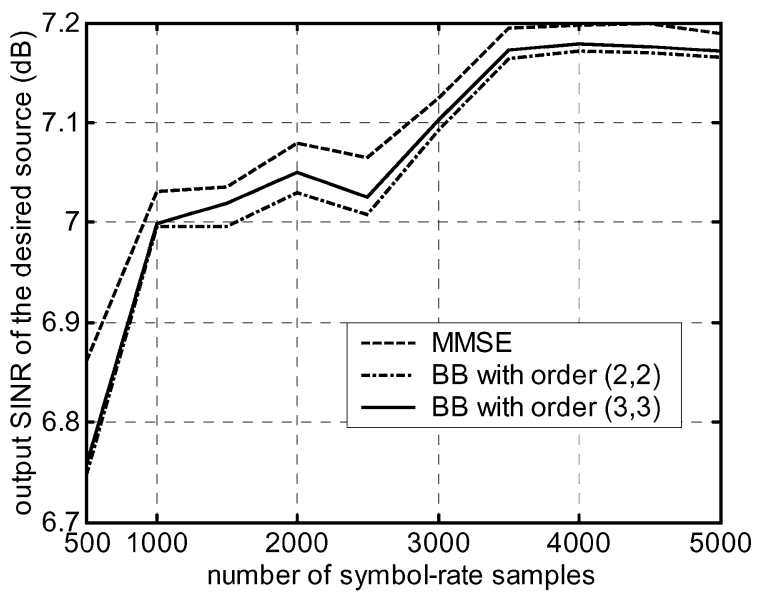

(b) under I2

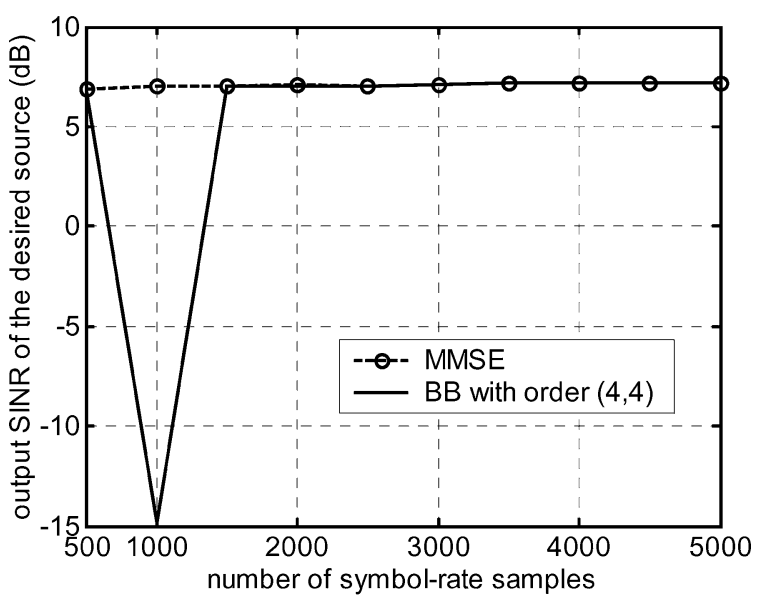

(c) under I2

Fig. 3. Effects of number of samples on performance.

is $10 \mathrm{~dB}$. It is seen that the convergent beam patterns corresponding to both initial conditions are very close to that of the optimal nonblind MMSE beamformer.

As a conclusion of the simulation results depicted in Figs. $1-4$, we see that the proposed beamforming algorithm is robust and effective. The simulation results demonstrated that the cumulant orders, i.e., $(2,2),(3,3)$, and $(4,4)$ are applicable, and using the order $(2,2)$ leads to lower computations. Although using higher order comulants is helpful to improve the convergence rate, the algorithm will be more sensitive to the

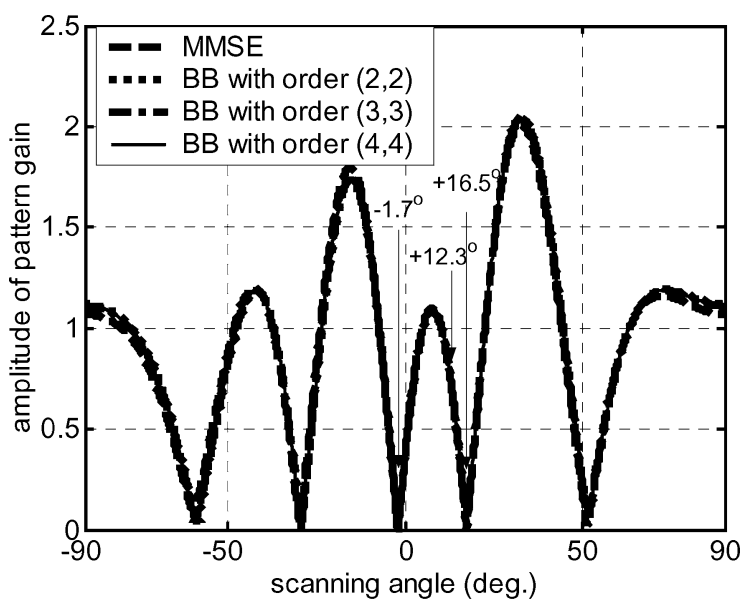

(a) under I1

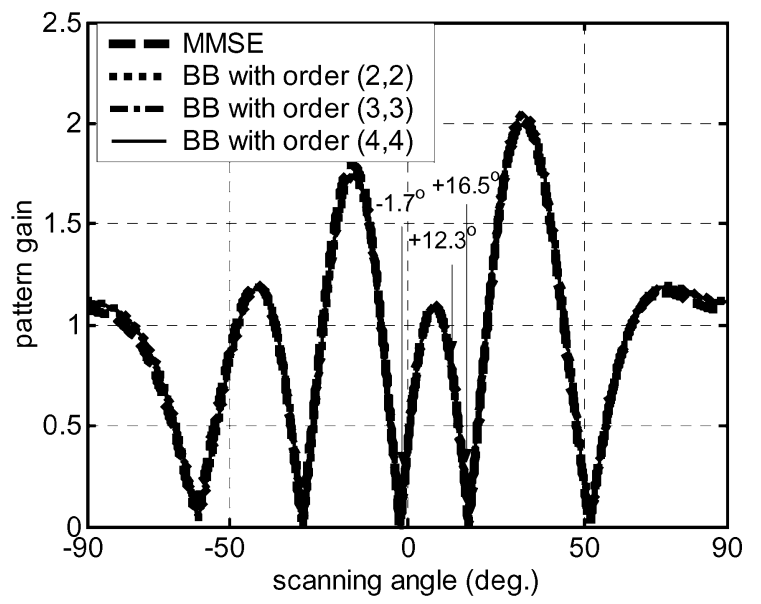

(b) under 12

Fig. 4. Convergent patterns.

accuracy of cumulant estimation when a higher order is used. Regarding the initial array patterns, the simulation results show that the algorithm under the omnidirectional pattern by $\mathrm{I} 2$ is robust in most situations, even when there are small differences in power between the desired source signal and the undesired source signals.

\section{CONCLUSIONS}

In this paper, we have presented the super-exponential blind adaptive beamforming algorithm, which is an extension from the super-exponential blind deconvolution theory and the inverse filter criteria. This extension theoretically considers the presence of noise such that the proposed beamforming algorithm is applicable, regardless of whether the number of independent sources exceeds the system degrees of freedom or not. We have also proved two special conditions under which the performance of the proposed blind algorithm approaches that of the optimal nonblind MMSE beamformer. Simulation results have shown that the proposed algorithm is effective and robust, even when the initial weight vector corresponding to the omnidirectional pattern is used. It should be noted that using the higher order cumulants increases the computational complexity and the sensitivity to the estimation accuracy of the cumulants and decreases the robustness to the model assumptions. 
APPENDIX A

PROOF OF THEOREM 1

\section{A. Proof}

When $p+q \geq 2$, (20) can be expressed as $s_{n}^{(k)}$

$$
=\frac{\left(\frac{\left|\beta_{n_{0}}\right|}{\left|\beta_{n}\right|}\right)^{1 /(p+q-1)}\left(\frac{\left|a_{n} \| \beta_{n}\right|^{1 /(p+q-1)}}{\left|a_{n_{0}} \| \beta_{n_{0}}\right|^{1 /(p+q-1)}}\right)^{(p+q)^{k} j(p-q)^{k} \varphi_{n}+j \psi_{n} \sum_{\models 0}^{k-1}(p-q)^{l}}}{\sqrt{1+\sum_{\substack{\ell=1 \\ l \neq n_{0}}}^{P+N}\left(\frac{\left|\beta_{n_{0}}\right|}{|\beta|}\right)^{2 /(p+q-1)}\left(\frac{\left.\left|a_{l} \|\right| \beta_{l}\right|^{1 /(p+q-1)}}{\left|a_{n_{0}}\right|\left|\beta_{n_{0}}\right|^{1 /(p+q-1)}}\right)^{2(p+q)^{k}}}} .
$$

Under the condition (22), that is

$$
\frac{\left|a_{n}\right|\left|\beta_{n}\right|^{1 /(p+q-1)}}{\left|a_{n_{0}}\right|\left|\beta_{n_{0}}\right|^{1 /(p+q-1)}}<1, n=1, \ldots, P+N, n \neq n_{0}
$$

where $\beta_{n}=\left|\beta_{n}\right| e^{j \psi_{n}},\left|\beta_{n}\right|<\infty, n=1, \ldots, P+N$, and the nominator of $\left|s_{n}^{(k)}\right|$ super-exponentially $\left((p+q)^{k}\right)$ converges to zero as $k \rightarrow \infty$, i.e., as in (A3) and (A4), shown at the bottom of the page. Therefore, as $k \rightarrow \infty$

$$
\begin{aligned}
& \left|s_{n}^{(k)}\right| \rightarrow 0, n=1, \ldots, P+N, n \neq n_{0} \\
& \left|s_{n_{0}}^{(k)}\right| \rightarrow 1 .
\end{aligned}
$$

Thus, the theorem has been proved.

APPENDIX B

PROOF OF THEOREM 2

\section{A. Proof}

By substituting (27) and (25) into (23) and by (2), the gain vector at the iteration $k$ can be simplified as

$$
\begin{aligned}
\mathbf{s}^{(k)}= & {\left[\begin{array}{c}
s_{1}^{(k)} \\
\vdots \\
s_{P+N}^{(k)}
\end{array}\right]=\frac{1}{\Delta^{(k-1)}} \mathbf{H}^{T}\left(\mathbf{R}^{T}\right)^{-1} \mathbf{H}^{*} \mathbf{g}^{(k-1)} } \\
= & \frac{1}{\Delta^{(k-1)}}\left[\begin{array}{cc}
\overline{\mathbf{A}}^{T}(\Theta)\left(\mathbf{R}^{T}\right)^{-1} \overline{\mathbf{A}}^{*}(\Theta) & \sigma_{\varpi} \overline{\mathbf{A}}^{T}(\Theta)\left(\mathbf{R}^{T}\right)^{-1} \\
\sigma_{\varpi}\left(\mathbf{R}^{T}\right)^{-1} \overline{\mathbf{A}}^{*}(\Theta) & \sigma_{\varpi}^{2}\left(\mathbf{R}^{T}\right)^{-1}
\end{array}\right] \\
& \times\left[\begin{array}{c}
\beta_{1}\left(s_{1}^{(k-1)}\right)^{p}\left(s_{1}^{(k-1) *}\right)^{q} \\
\vdots \\
\beta_{P+N}\left(s_{P+N}^{(k-1)}\right)^{p}\left(s_{P+N}^{(k-1) *}\right)^{q}
\end{array}\right]
\end{aligned}
$$

where

$$
\begin{aligned}
\Delta^{(k-1)} & =\sqrt{\left(\mathbf{d}^{(k-1)}\right)^{H}\left(\mathbf{R}^{T}\right)^{-1} \mathbf{d}^{(k-1)}} \\
& =\left\|\mathbf{H}^{T}\left(\mathbf{H}^{*} \mathbf{H}^{T}\right)^{-1} \mathbf{H}^{*} \mathbf{g}^{(k-1)}\right\| .
\end{aligned}
$$

Because the cumulants of Gaussian noises are equal to zeros when $p+q \geq 2$ and $P \leq N$, (B1) thus becomes

$$
\begin{aligned}
\mathbf{s}^{(k)}=\frac{1}{\Delta^{(k-1)}}\left[\begin{array}{c}
\overline{\mathbf{A}}^{T}(\Theta)\left(\mathbf{R}^{T}\right)^{-1} \overline{\mathbf{A}}^{*}(\Theta) \\
\sigma_{\varpi}\left(\mathbf{R}^{T}\right)^{-1} \overline{\mathbf{A}}^{*}(\Theta)
\end{array}\right] \\
\times\left[\begin{array}{c}
\beta_{1}\left(s_{1}^{(k-1)}\right)^{p}\left(s_{1}^{(k-1) *}\right)^{q} \\
\vdots \\
\beta_{P}\left(s_{P}^{(-1)}\right)^{\dot{p}}\left(s_{P}^{(k-1) *}\right)^{q}
\end{array}\right] .
\end{aligned}
$$

With the use of Sherman-Morrison-Woodbury formula [25], under assumption $\mathrm{A} 3$, the inversion of $\mathbf{R}^{T}$ can be written as

$$
\begin{aligned}
\left(\mathbf{R}^{T}\right)^{-1}= & \left(\overline{\mathbf{A}}^{*}(\Theta) \overline{\mathbf{A}}^{T}(\Theta)+\sigma_{\varpi}^{2} \mathbf{I}\right)^{-1} \\
= & \frac{1}{\sigma_{\varpi}^{2}} \mathbf{I}-\frac{1}{\sigma_{\varpi}^{4}} \overline{\mathbf{A}}^{*}(\Theta)\left(\mathbf{I}+\frac{\overline{\mathbf{A}}^{T}(\Theta) \overline{\mathbf{A}}^{*}(\Theta)}{\sigma_{\varpi}^{2}}\right)^{-1} \overline{\mathbf{A}}^{T}(\Theta) \\
= & \frac{1}{\sigma_{\varpi}^{2}} \mathbf{I}-\frac{1}{\sigma_{\varpi}^{4}} \overline{\mathbf{A}}^{*}(\Theta)\left(\frac{\overline{\mathbf{A}}^{T}(\Theta) \overline{\mathbf{A}}^{*}(\Theta)}{\sigma_{\varpi}^{2}}\right)^{-1} \\
& \times\left(\mathbf{I}+\left(\frac{\overline{\mathbf{A}}^{T}(\Theta) \overline{\mathbf{A}}^{*}(\Theta)}{\sigma_{\varpi}^{2}}\right)^{-1}\right)^{-1} \overline{\mathbf{A}}^{T}(\Theta) . \quad(\mathrm{B} 4
\end{aligned}
$$

Therefore

$$
\begin{aligned}
\overline{\mathbf{A}}^{T}(\Theta)\left(\mathbf{R}^{T}\right)^{-1} \overline{\mathbf{A}}^{*}(\Theta)= & \frac{\overline{\mathbf{A}}^{T}(\Theta) \overline{\mathbf{A}}^{*}(\Theta)}{\sigma_{\varpi}^{2}} \\
& -\frac{1}{\sigma_{\varpi}^{4}} \overline{\mathbf{A}}^{T}(\Theta) \overline{\mathbf{A}}^{*}(\Theta)\left(\frac{\overline{\mathbf{A}}^{T}(\Theta) \overline{\mathbf{A}}^{*}(\Theta)}{\sigma_{\varpi}^{2}}\right)^{-1} \\
& \times\left(\mathbf{I}+\left(\frac{\overline{\mathbf{A}}^{T}(\Theta) \overline{\mathbf{A}}^{*}(\Theta)}{\sigma_{\varpi}^{2}}\right)^{-1}\right)^{-1} \overline{\mathbf{A}}^{T}(\Theta) \overline{\mathbf{A}}^{*}(\Theta) \\
= & \left(\mathbf{I}+\left(\frac{\overline{\mathbf{A}}^{T}(\Theta) \overline{\mathbf{A}}^{*}(\Theta)}{\sigma_{\varpi}^{2}}\right)^{-1}\right)^{-1} \cdot \text { (B5) }
\end{aligned}
$$

Similarly

$$
\begin{aligned}
\sigma_{\varpi}\left(\mathbf{R}^{T}\right)^{-1} \overline{\mathbf{A}}^{*}(\Theta)= & \frac{\overline{\mathbf{A}}^{*}(\Theta)}{\sigma_{\varpi}} \\
& -\frac{1}{\sigma_{\varpi}^{3}} \overline{\mathbf{A}}^{*}(\Theta)\left(\mathbf{I}+\left(\frac{\overline{\mathbf{A}}^{T}(\Theta) \overline{\mathbf{A}}^{*}(\Theta)}{\sigma_{\varpi}^{2}}\right)^{-1}\right)^{-1} \\
& \times\left(\frac{\overline{\mathbf{A}}^{T}(\Theta) \overline{\mathbf{A}}^{*}(\Theta)}{\sigma_{\varpi}^{2}}\right)^{-1} \overline{\mathbf{A}}^{T}(\Theta) \overline{\mathbf{A}}^{*}(\Theta) \\
= & \frac{\overline{\mathbf{A}}^{*}(\Theta)}{\sigma_{\varpi}}\left(\mathbf{I}+\frac{\overline{\mathbf{A}}^{T}(\Theta) \overline{\mathbf{A}}^{*}(\Theta)}{\sigma_{\varpi}^{2}}\right)^{-1} \cdot \quad \text { (B6) }
\end{aligned}
$$

$$
\begin{aligned}
& \left(\frac{\left|\beta_{n_{0}}\right|}{\left|\beta_{n}\right|}\right)^{1 /(p+q-1)}\left(\frac{\left|a_{n}\right|\left|\beta_{n}\right|^{1 /(p+q-1)}}{\left|a_{n_{0}}\right|\left|\beta_{n_{0}}\right|^{1 /(p+q-1)}}\right)^{(p+q)^{k}} \rightarrow 0 \\
& \sqrt{1+\sum_{\substack{l=1 \\
l \neq n_{0}}}^{P+N}\left(\frac{\left|\beta_{n_{0}}\right|}{\left|\beta_{l}\right|}\right)^{2 /(p+q-1)}\left(\frac{\left|a_{l}\right|\left|\beta_{l}\right|^{1 /(p+q-1)}}{\left|a_{n_{0}}\right|\left|\beta_{n_{0}}\right|^{1 /(p+q-1)}}\right)^{2(p+q)^{k}}} \stackrel{k \rightarrow \infty}{\rightarrow} 1 .
\end{aligned}
$$


When $\sigma_{b_{i}}^{2} / \sigma_{\varpi}^{2} \rightarrow+\infty, i=1, \ldots, P$, (B5) and (B6) approach the following results:

$$
\begin{aligned}
\overline{\mathbf{A}}^{T}(\Theta)\left(\mathbf{R}^{T}\right)^{-1} \overline{\mathbf{A}}^{*}(\Theta) & \rightarrow \mathbf{I} \\
\sigma_{\varpi}\left(\mathbf{R}^{T}\right)^{-1} \overline{\mathbf{A}}^{*}(\Theta) & \rightarrow \mathbf{0}
\end{aligned}
$$

and substituting (B7) and (B8) into (B3), we have

$$
\mathbf{s}^{(k)}=\frac{1}{\Delta^{(k-1)}}\left[\begin{array}{c}
\beta_{1}\left(s_{1}^{(k-1)}\right)^{p}\left(s_{1}^{(k-1) *}\right)^{q} \\
\vdots \\
\beta_{P}\left(s_{P}^{(k-1)}\right)_{\mathbf{0}}^{p}\left(s_{P}^{(k-1) *}\right)^{q}
\end{array}\right]
$$

where

$$
\Delta^{(k-1)}=\sqrt{\sum_{n=1}^{P}\left|\beta_{n}\left(s_{n}^{(k-1)}\right)^{p}\left(s_{n}^{(k-1) *}\right)^{q}\right|^{2}} .
$$

Because (B9) actually corresponds to a special case of (18) considered in Theorem 1, applying the conclusion of Theorem 1 proves Theorem 2 .

APPENDIX C

\section{PROOF OF THEOREM 3}

\section{A. Proof}

Similar to the proof of Theorem 2, when $P \leq N$ and the associated noises are Gaussian distributed, we rewrite the iterative gain vector of (B3) as (C1), shown at the bottom of the page. Define

$$
\begin{aligned}
\overline{\mathbf{A}}_{i}(\Theta) & =\left[\sigma_{b_{1}} \mathbf{a}\left(\theta_{1}\right), \ldots, \sigma_{b_{i-1}} \mathbf{a}\left(\theta_{i-1}\right), \sigma_{b_{i+1}} \mathbf{a}\left(\theta_{i+1}\right), \ldots, \sigma_{b_{P}} \mathbf{a}\left(\theta_{P}\right)\right] \\
\mathbf{R}_{i} & =\overline{\mathbf{A}}_{i}(\Theta) \overline{\mathbf{A}}_{i}^{H}(\Theta)+\sigma_{\varpi}^{2} \mathbf{I} .
\end{aligned}
$$

The condition that $\mathbf{a}^{T}\left(\theta_{n}\right) \mathbf{a}^{*}\left(\theta_{n_{0}}\right)=0, n=1, \ldots, P, n \neq n_{0}$ implies

$$
\overline{\mathbf{A}}_{n_{0}}^{T}(\Theta) \mathbf{a}^{*}\left(\theta_{n_{0}}\right)=\mathbf{0} .
$$

Because of (6), $\mathbf{R}$ can be expressed as

$$
\mathbf{R}=\mathbf{R}_{n_{0}}+\sigma_{b_{n_{0}}} \mathbf{a}\left(\theta_{n_{0}}\right)\left(\sigma_{b_{n_{0}}} \mathbf{a}\left(\theta_{n_{0}}\right)\right)^{H} .
$$

According to the Sherman-Morrison-Woodbury formula [25], the inversion of $\mathbf{R}$ can be denoted as

$$
\left(\mathbf{R}^{T}\right)^{-1}=\left(\mathbf{R}_{n_{0}}^{T}\right)^{-1}-\frac{\left(\mathbf{R}_{n_{0}}^{T}\right)^{-1} \sigma_{b_{n_{0}}} \mathbf{a}^{*}\left(\theta_{n_{0}}\right) \sigma_{b_{n_{0}}} \mathbf{a}^{T}\left(\theta_{n_{0}}\right)\left(\mathbf{R}_{n_{0}}^{T}\right)^{-1}}{1+\sigma_{b_{n_{0}}}^{2} \mathbf{a}^{T}\left(\theta_{n_{0}}\right)\left(\mathbf{R}_{n_{0}}^{T}\right)^{-1} \mathbf{a}^{*}\left(\theta_{n_{0}}\right)} \text {. }
$$

Similarly, by employing the Sherman-Morrison-Woodbury formula to $\left(\mathbf{R}_{n_{0}}^{T}\right)^{-1}$ and with (C4), we obtain

$$
\left(\mathbf{R}_{n_{0}}^{T}\right)^{-1} \mathbf{a}^{*}\left(\theta_{n_{0}}\right)=\frac{1}{\sigma_{\varpi}^{2}} \mathbf{a}^{*}\left(\theta_{n_{0}}\right) .
$$

Therefore

$$
\left(\mathbf{R}^{T}\right)^{-1} \mathbf{a}^{*}\left(\theta_{n_{0}}\right)=\frac{\frac{\mathbf{a}^{*}\left(\theta_{n_{0}}\right)}{\sigma_{\varpi}^{2}}}{1+\frac{\left\|\mathbf{a}\left(\theta_{n_{0}}\right)\right\|^{2} \sigma_{b_{n_{0}}}^{2}}{\sigma_{\varpi}^{2}}} .
$$

Substituting (C8) into (C1) and by (C4), we have (C9), shown at the bottom of the next page, where

$$
\begin{aligned}
& \Delta^{(k-1)} \\
= & \left(\left|\mu_{0} \beta_{n_{0}}\left(s_{n_{0}}^{(k-1)}\right)^{p}\left(s_{n_{0}}^{(k-1) *}\right)^{q}\right|^{2}\right. \\
& +\sum_{\substack{l=1 \\
l \neq n_{0}}}^{P}\left|\sum_{\substack{i=1 \\
i \neq n_{0}}}^{P} \sigma_{b_{l}} \sigma_{b_{i}} \mathbf{a}^{T}\left(\theta_{l}\right)\left(\mathbf{R}^{T}\right)^{-1} \mathbf{a}^{*}\left(\theta_{i}\right) \beta_{i}\left(s_{i}^{(k-1)}\right)^{p}\left(s_{i}^{(k-1) *}\right)^{q}\right|^{2} \\
& \left.+\left\|\sum_{i=1}^{P} \sigma_{\varpi} \sigma_{b_{i}}\left(\mathbf{R}^{T}\right)^{-1} \mathbf{a}^{*}\left(\theta_{i}\right) \beta_{i}\left(s_{i}^{(k-1)}\right)^{p}\left(s_{i}^{(k-1) *}\right)^{q}\right\|^{2}\right)^{1 / 2}
\end{aligned}
$$

$$
=\frac{\frac{\left\|\mathbf{a}\left(\theta_{n_{0}}\right)\right\|^{2} \sigma_{b_{n_{0}}}^{2}}{\sigma_{\varpi}^{2}}}{1+\frac{\left\|\mathbf{a}\left(\theta_{n_{0}}\right)\right\|^{2} \sigma_{b_{n_{0}}}^{2}}{\sigma_{\varpi}^{2}}}<1
$$

According to (C9), the following inequalities stand:

$$
\begin{aligned}
& \left|s_{n}^{(k)}\right| \\
\leq & \frac{1}{\Delta^{(k-1)}} \sum_{\substack{i=1 \\
i \neq n_{0}}}^{P}\left|\sigma_{b_{n}} \sigma_{b_{i}} \mathbf{a}^{T}\left(\theta_{n}\right)\left(\mathbf{R}^{T}\right)^{-1} \mathbf{a}^{*}\left(\theta_{i}\right)\right|\left|\beta_{i}\right|\left|s_{i}^{(k-1)}\right|^{p+q} \\
& n=1, \ldots, P, n \neq n_{0} \\
= & \left.\frac{1}{\Delta^{(k-1)}} \mu_{0}\left|\beta_{n_{0}}\right|\left|s_{n_{0}}^{(k-1)}\right|\right|^{p+q} \\
& \left|s_{P+l}^{(k)}\right| \\
= & \frac{1}{\Delta^{(k-1)}} \sum_{i=1}^{P}\left(\sigma_{\varpi} \sigma_{b_{i}}\left(\mathbf{R}^{T}\right)^{-1} \mathbf{a}^{*}\left(\theta_{i}\right)\right)_{l} \beta_{i}\left(s_{i}^{(k-1)}\right)^{p}\left(s_{i}^{(k-1) *}\right)^{q} \\
& l=1, \ldots, N .
\end{aligned}
$$

It can be seen that $s_{P+l}^{(k)}, l=1, \ldots, N$ depends on $s_{n}^{(k)}$, $n=1, \ldots, P$, whereas we are only concerned with the iterative behavior of $s_{n}^{(k)}, n=1, \ldots, P$. Let $\left|s_{m_{0}}^{(0)}\right|=$ $\max \left\{\left|s_{n}^{(0)}\right|, n=1, \ldots, P, n \neq n_{0}\right\}$, i.e., for $n=1, \ldots, P$

$$
\left|s_{n}^{(0)}\right| \leq\left|s_{m_{0}}^{(0)}\right| \text {. }
$$

(C13)

$$
\mathbf{s}^{(k)}=\frac{1}{\Delta^{(k-1)}}\left[\begin{array}{ccc}
\sigma_{b_{1}}^{2} \mathbf{a}^{T}\left(\theta_{1}\right)\left(\mathbf{R}^{T}\right)^{-1} \mathbf{a}^{*}\left(\theta_{1}\right) & \cdots & \sigma_{b_{1}} \sigma_{b_{P}} \mathbf{a}^{T}\left(\theta_{1}\right)\left(\mathbf{R}^{T}\right)^{-1} \mathbf{a}^{*}\left(\theta_{P}\right) \\
\vdots & \ddots & \vdots \\
\sigma_{b_{P}} \sigma_{b_{1}} \mathbf{a}^{T}\left(\theta_{P}\right)\left(\mathbf{R}^{T}\right)^{-1} \mathbf{a}^{*}\left(\theta_{1}\right) & \cdots & \sigma_{b_{P}}^{2} \mathbf{a}^{T}\left(\theta_{P}\right)\left(\mathbf{R}^{T}\right)^{-1} \mathbf{a}^{*}\left(\theta_{P}\right) \\
\sigma_{b_{1}} \sigma_{\varpi}\left(\mathbf{R}^{T}\right)^{-1} \mathbf{a}^{*}\left(\theta_{1}\right) & \cdots & \sigma_{b_{P}} \sigma_{\varpi}\left(\mathbf{R}^{T}\right)^{-1} \mathbf{a}^{*}\left(\theta_{P}\right)
\end{array}\right] \times\left[\begin{array}{c}
\beta_{1}\left(s_{1}^{(k-1)}\right)^{p}\left(s_{1}^{(k-1) *}\right)^{q} \\
\vdots \\
\beta_{P}\left(s_{P}^{(k-1)}\right)^{p}\left(s_{P}^{(k-1) *}\right)^{q}
\end{array}\right] .
$$


By (C13), (C12a) and (C12b), the following relations can be easily inferred:

$$
\begin{aligned}
& \left|s_{n}^{(1)}\right| \\
& \leq \frac{1}{\Delta^{(0)}}\left|s_{m_{0}}^{(0)}\right|^{p+q} \sum_{\substack{i=1 \\
i \neq n_{0}}}^{P}\left|\sigma_{b_{n}} \sigma_{b_{2}} \mathbf{a}^{T}\left(\theta_{n}\right)\left(\mathbf{R}^{T}\right)^{-1} \mathbf{a}^{*}\left(\theta_{i}\right)\right|\left|\beta_{i}\right| \\
& n=1, \ldots, P, n \neq n_{0} \\
& \left|s_{n_{0}}^{(1)}\right| \\
& =\frac{1}{\Delta^{(0)}} \mu_{0}\left|\beta_{n_{0}}\right|\left|s_{n_{0}}^{(0)}\right|^{p+q} \\
& \begin{aligned}
& \Delta^{(0)} \\
= & \left|\beta_{n_{0}}\right|^{2}\left|s_{n_{0}}^{(0)}\right|^{2(p+q)}
\end{aligned} \\
& +\sum_{\substack{l=1 \\
l \neq n_{0}}}^{P}\left|\sum_{\substack{i=1 \\
i \neq n_{0}}}^{P} \sigma_{b_{l}} \sigma_{b_{\imath}} \mathbf{a}^{T}\left(\theta_{l}\right)\left(\mathbf{R}^{T}\right)^{-1} \mathbf{a}^{*}\left(\theta_{i}\right) \beta_{i}\left(s_{i}^{(0)}\right)^{p}\left(s_{i}^{(0) *}\right)^{q}\right|^{2} \\
& \left.+\left\|\sum_{i=1}^{P} \sigma_{\varpi} \sigma_{b_{i}}\left(\mathbf{R}^{T}\right)^{-1} \mathbf{a}^{*}\left(\theta_{i}\right) \beta_{i}\left(s_{i}^{(0)}\right)^{p}\left(s_{i}^{(0) *}\right)^{q}\right\|^{2}\right)^{1 / 2} . \\
& \widetilde{\beta}_{n}=\sum_{\substack{i=1 \\
i \neq n_{0}}}^{P}\left|\sigma_{b_{n}} \sigma_{b_{i}} \mathbf{a}^{T}\left(\theta_{n}\right)\left(\mathbf{R}^{T}\right)^{-1} \mathbf{a}^{*}\left(\theta_{i}\right)\right|\left|\beta_{i}\right| \\
& n=1, \ldots, P, n \neq n_{0} \\
& \widetilde{\beta}_{n_{0}}=\mu_{0}\left|\beta_{n_{0}}\right| \\
& \widetilde{\beta}_{r_{0}}=\max \left\{\widetilde{\beta}_{n}, n=1, \ldots, P, n \neq n_{0}\right\}, 1 \leq r_{0} \leq P(\mathrm{C} 16 \mathrm{c})
\end{aligned}
$$

so we have

$$
\left|s_{n}^{(1)}\right| \leq \frac{1}{\Delta^{(0)}} \widetilde{\beta}_{n}\left|s_{m_{0}}^{(0)}\right|^{p+q} \leq \frac{1}{\Delta^{(0)}} \widetilde{\beta}_{r_{0}}\left|s_{m_{0}}^{(0)}\right|^{p+q}, n=1, \ldots, P .
$$

$$
\begin{aligned}
\left|s_{n}^{(2)}\right| & \leq \frac{1}{\Delta^{(1)}} \sum_{\substack{i=1 \\
i \neq n_{0}}}^{P}\left|\sigma_{b_{n}} \sigma_{b_{i}} \mathbf{a}^{T}\left(\theta_{n}\right)\left(\mathbf{R}^{T}\right)^{-1} \mathbf{a}^{*}\left(\theta_{i}\right)\right|\left|\beta_{i}\right|\left|s_{i}^{(1)}\right|^{p+q} \\
& \leq \frac{1}{\Delta^{(1)}} \widetilde{\beta}_{n}\left(\widetilde{\beta}_{r_{0}}\left|s_{m_{0}}^{(0)}\right|^{p+q}\right)^{p+q} \\
& \leq \frac{1}{\Delta^{(1)}} \widetilde{\beta}_{r_{0}}^{p+q+1}\left|s_{m_{0}}^{(0)}\right|^{(p+q)^{2}}, n=1, \ldots, P, n \neq n_{0}(\mathrm{C} 18 \mathrm{a}) \\
\left|s_{n_{0}}^{(2)}\right| & =\frac{1}{\Delta^{(1)}} \widetilde{\beta}_{n_{0}}^{p+q+1}\left|s_{n_{0}}^{(0)}\right|^{(p+q)^{2}} \leq \frac{1}{\Delta^{(1)}} \widetilde{\beta}_{r_{0}}^{p+q+1}\left|s_{m_{0}}^{(0)}\right|^{(p+q)^{2}} .
\end{aligned}
$$

Repeatedly, we have the iterative equations

$$
\begin{aligned}
& \left|s_{n_{0}}^{(k)}\right| \\
& =\frac{1}{\Delta^{(k-1)}} \widetilde{\beta}_{n_{0}}^{\left((p+q)^{k}-1\right) /(p+q-1)}\left|s_{n_{0}}^{(0)}\right|^{(p+q)^{k}} \\
& \leq \frac{1}{\Delta^{(k-1)}} \widetilde{\beta}_{r_{0}}^{\left((p+q)^{k}-1\right) /(p+q-1)}\left|s_{m_{0}}^{(0)}\right|^{(p+q)^{k}} \\
& \left|s_{n}^{(k)}\right| \\
& \leq \frac{1}{\Delta^{(k-1)}} \widetilde{\beta}_{r_{0}}^{\left((p+q)^{k}-1\right) /(p+q-1)}\left|s_{m_{0}}^{(0)}\right|^{(p+q)^{k}} \\
& n=1, \ldots, P+N, n \neq n_{0} \\
& \Delta^{(k-1)} \\
& =\left(\widetilde{\beta}_{n_{0}}^{2 \times\left((p+q)^{k}-1\right) /(p+q-1)}\left|s_{n_{0}}^{(0)}\right|^{2(p+q)^{k}}\right. \\
& +\sum_{\substack{l=1 \\
l \neq n_{0}}}^{P}\left|\sum_{\substack{i=1 \\
i \neq n_{0}}}^{P} \sigma_{b_{l}} \sigma_{b_{i}} \mathbf{a}^{T}\left(\theta_{l}\right)\left(\mathbf{R}^{T}\right)^{-1} \mathbf{a}^{*}\left(\theta_{i}\right) \beta_{i}\left(s_{i}^{(k-1)}\right)^{p}\left(s_{i}^{(k-1) *}\right)^{q}\right|^{2} \\
& \left.+\left\|\sum_{i=1}^{P} \sigma_{\varpi} \sigma_{b_{i}}\left(\mathbf{R}^{T}\right)^{-1} \mathbf{a}^{*}\left(\theta_{i}\right) \beta_{i}\left(s_{i}^{(k-1)}\right)^{p}\left(s_{i}^{(k-1) *}\right)^{q}\right\|^{2}\right)^{1 / 2} .
\end{aligned}
$$

(C19c)

$$
\mathbf{s}^{(k)}=\frac{1}{\Delta^{(k-1)}}\left[\begin{array}{c}
\sum_{\substack{i=1 \\
i \neq n_{0}}}^{P} \sigma_{b_{1}} \sigma_{b_{i}} \mathbf{a}^{T}\left(\theta_{1}\right)\left(\mathbf{R}^{T}\right)^{-1} \mathbf{a}^{*}\left(\theta_{i}\right) \beta_{i}\left(s_{i}^{(k-1)}\right)^{p}\left(s_{i}^{(k-1) *}\right)^{q} \\
\vdots \\
\sum_{\substack{i=1 \\
i \neq n_{0}}}^{P} \sigma_{b_{n_{0}-1}} \sigma_{b_{i}} \mathbf{a}^{T}\left(\theta_{n_{0}-1}\right)\left(\mathbf{R}^{T}\right)^{-1} \mathbf{a}^{*}\left(\theta_{i}\right) \beta_{i}\left(s_{i}^{(k-1)}\right)^{p}\left(s_{i}^{(k-1) *}\right)^{q} \\
\sum_{\substack{i=1 \\
i \neq n_{0}}}^{P} \sigma_{b_{n_{0}+1} \sigma_{b_{i}}} \mathbf{a}^{T}\left(\theta_{n_{0}+1}\right)\left(\mathbf{R}^{T}\right)^{-1} \mathbf{a}^{*}\left(\theta_{i}\right) \beta_{i}\left(s_{i}^{(k-1)}\right)^{p}\left(s_{i}^{(k-1) *}\right)^{q} \\
\sum_{\substack{i=1 \\
i \neq n_{0}}}^{P} \sigma_{b_{P}} \sigma_{b_{i}} \mathbf{a}^{T}\left(\theta_{P}\right)\left(\mathbf{R}^{T}\right)^{-1} \mathbf{a}^{*}\left(\theta_{i}\right) \beta_{i}\left(s_{i}^{(k-1)}\right)^{p}\left(s_{i}^{(k-1) *}\right)^{q} \\
\sum_{i=1}^{P} \sigma_{\varpi} \sigma_{b_{i}}\left(\mathbf{R}^{T}\right)^{-1} \mathbf{a}^{*}\left(\theta_{i}\right) \beta_{i}\left(s_{i}^{(k-1)}\right)^{p}\left(s_{i}^{(k-1) *}\right)^{q}
\end{array}\right]
$$


Therefore

$$
\begin{array}{r}
\left|s_{n}^{(k)}\right| \leq\left(\frac{\widetilde{\beta}_{n_{0}}}{\widetilde{\beta}_{r_{0}}}\right)^{1 /(p+q-1)}\left(\frac{\widetilde{\beta}_{r_{0}}^{1 /(p+q-1)}\left|s_{m_{0}}^{(0)}\right|}{\widetilde{\beta}_{n_{0}}^{1 /(p+q-1)}\left|s_{n_{0}}^{(0)}\right|}\right)^{(p+q)^{k}} \\
n=1, \ldots, P, n \neq n_{0} .
\end{array}
$$

When $\widetilde{\beta}_{n_{0}}^{1 /(p+q-1)}\left|s_{n_{0}}^{(0)}\right|>\widetilde{\beta}_{r_{0}}^{1 /(p+q-1)} s_{m_{0}}^{(0)}$, as $k \rightarrow \infty$, the following term will super-exponentially converges to zero:

$$
\left(\frac{\widetilde{\beta}_{r_{0}}^{1 /(p+q-1)}\left|s_{m_{0}}^{(0)}\right|}{\widetilde{\beta}_{n_{0}}^{1 /(p+q-1)}\left|s_{n_{0}}^{(0)}\right|}\right)^{\stackrel{(p+q)^{k}}{\rightarrow} 0} \stackrel{k \rightarrow \infty}{\rightarrow} 0 .
$$

Because of (C8), (C21) implies that

$$
\begin{aligned}
& \left|s_{n}^{(k)}\right| \stackrel{k \rightarrow \infty}{\rightarrow} 0, n=1, \ldots, P, n \neq n_{0} \\
& \left|s_{n_{0}}^{(k)}\right| \stackrel{k \rightarrow \infty}{\rightarrow} \frac{1}{\sqrt{1+\frac{1}{\frac{\left\|\mathbf{a}\left(\theta_{n_{0}}\right)\right\|^{2} \sigma_{b_{n_{0}}^{2}}^{2}}{\sigma_{\varpi}^{2}}}}} .
\end{aligned}
$$

Therefore, from (C9), we have

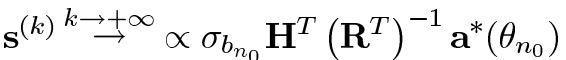

$$
\begin{aligned}
& =\frac{\frac{\mathbf{H}^{T} \mathbf{a}^{*}\left(\theta_{n_{0}}\right) \sigma_{b_{n_{0}}}}{\sigma_{\varpi}^{2}}}{1+\frac{\left\|\mathbf{a}\left(\theta_{n_{0}}\right)\right\|^{2} \sigma_{b_{n_{0}}}^{2}}{\sigma_{\varpi}^{2}}} .
\end{aligned}
$$

It can be easily seen from $(\mathrm{C} 23)$ that as $k$ increases, $\mathbf{s}^{(k)}$ approaches the gain vector of the optimal nonblind MMSE beamformer up to a complex rotating phase factor. Thus, Theorem 3 has been proved.

\section{APPENDIX D}

PROOF OF THEOREM 4

\section{A. Proof}

When the super-exponential iterative algorithm converges, (35a)-(35c) can be written as

$$
\begin{aligned}
\mathbf{c} & =\frac{\left(\mathbf{R}^{T}\right)^{-1} \mathbf{d}}{\sqrt{\mathbf{d}^{H}\left(\mathbf{R}^{T}\right)^{-1} \mathbf{d}}} \\
\mathbf{d} & =\operatorname{cum}\left(z(t): m ; z^{*}(t): m-1 ; \mathbf{x}^{*}(t)\right) \\
z(t) & =\mathbf{c}^{T} \mathbf{x}(t) .
\end{aligned}
$$

(D1a)

Substituting (D1a) into (59), we have

$$
\begin{aligned}
& \frac{\partial J_{2 m}(\mathbf{c})}{\partial \mathbf{c}}=m \times\left(\operatorname{sgn}\left(C_{m, m}\{z(t)\}\right)\right. \\
& \left.\times \operatorname{cum}\left\{z(t): m, z^{*}(t): m-1, \mathbf{x}^{*}(\mathrm{t})\right\}-\frac{\left|C_{m, m}\{z(t)\}\right|}{\sqrt{\mathbf{d}^{H}\left(\mathbf{R}^{T}\right)^{-1} \mathbf{d}}} \mathbf{d}\right)
\end{aligned}
$$

where $\mathbf{c}^{T} \mathbf{R} \mathbf{c}^{*}=1$ is used. Based on the linearity property of the cumulants [7] and with (D1b) and (D1c)

$$
\begin{aligned}
C_{m, m}\{z(t)\} & =\mathbf{c}^{H} \operatorname{cum}\left\{z(t): m, z^{*}(t): m-1, \mathbf{x}^{*}(\mathrm{t})\right\} \\
& =\mathbf{c}^{H} \mathbf{d}=\frac{\mathbf{d}^{H}\left(\mathbf{R}^{T}\right)^{-1} \mathbf{d}}{\sqrt{\mathbf{d}^{H}\left(\mathbf{R}^{T}\right)^{-1} \mathbf{d}}}>0
\end{aligned}
$$

which means

$$
\operatorname{sgn}\left(C_{m, m}\{z(t)\}\right)=+1 \text {. }
$$

Therefore

$$
\frac{\partial J_{2 m}(\mathbf{c})}{\partial \mathbf{c}}=\mathbf{0}
$$

and Theorem 4 has been proved.

\section{APPENDIX E}

Cumulants $\operatorname{cum}\left(z(t): m ; z^{*}(t): m\right)$ FOR $m=2,3,4$

Because analytical signals (complex envelope) in communications, radar, etc., are often of symmetrical distributions with odd order cumulants equal to zero, the following are formulae for computing $2 m$ th-order cumulants of $z(t)$ for $m=2,3$, and 4 as defined in [7]:

$$
\begin{aligned}
& \operatorname{cum}\left(z(t): 2 ; z^{*}(t): 2\right) \\
& =E\left(|z(t)|^{4}\right)-2\left(E\left(|z(t)|^{2}\right)\right)^{2} \\
& \operatorname{cum}\left(z(t): 2 ; z^{*}(t) ; \mathbf{x}^{*}(t)\right) \\
& =E\left(|z(t)|^{2} z(t) \mathbf{x}^{*}(t)\right)-2 E\left(|z(t)|^{2}\right) E\left(z(t) \mathbf{x}^{*}(t)\right) \\
& \operatorname{cum}\left(z(t): 3 ; z^{*}(t): 3\right) \\
& =E\left(|z(t)|^{6}\right)-9 E\left(|z(t)|^{4}\right) E\left(|z(t)|^{2}\right)+12\left(E\left(|z(t)|^{2}\right)\right)^{3} \\
& \operatorname{cum}\left(z(t): 3 ; z^{*}(t): 2 ; \mathbf{x}^{*}(t)\right) \\
& =E\left(|z(t)|^{4} z(t) \mathbf{x}^{*}(t)\right)-3 E\left(|z(t)|^{4}\right) E\left(z(t) \mathbf{x}^{*}(t)\right) \\
& -6 E\left(|z(t)|^{2}\right) E\left(|z(t)|^{2} z(t) \mathbf{x}^{*}(t)\right) \\
& +12\left(E\left(|z(t)|^{2}\right)\right)^{2} E\left(z(t) \mathbf{x}^{*}(t)\right) \\
& \operatorname{cum}\left(z(t): 4 ; z^{*}(t): 4\right) \\
& =E\left(|z(t)|^{8}\right)-16 E\left(|z(t)|^{6}\right) E\left(|z(t)|^{2}\right)-18\left(E\left(|z(t)|^{4}\right)\right)^{2} \\
& +144 E\left(|z(t)|^{4}\right)\left(E\left(|z(t)|^{2}\right)\right)^{2}-144\left(E\left(|z(t)|^{2}\right)\right)^{4} \\
& \operatorname{cum}\left(z(t): 4 ; z^{*}(t): 3 ; \mathbf{x}^{*}(t)\right) \\
& =E\left(|z(t)|^{6} z(t) \mathbf{x}^{*}(t)\right)-4 E\left(|z(t)|^{6}\right) E\left(z(t) \mathbf{x}^{*}(t)\right) \\
& -12 E\left(|z(t)|^{2}\right) E\left(|z(t)|^{4} z(t) \mathbf{x}^{*}(t)\right) \\
& -18 E\left(|z(t)|^{4}\right) E\left(|z(t)|^{2} z(t) \mathbf{x}^{*}(t)\right) \\
& +72 E\left(|z(t)|^{4}\right) E\left(|z(t)|^{2}\right) E\left(z(t) \mathbf{x}^{*}(t)\right) \\
& +72\left(E\left(|z(t)|^{2}\right)\right)^{2} E\left(|z(t)|^{2} z(t) \mathbf{x}^{*}(t)\right) \\
& -144\left(E\left(|z(t)|^{2}\right)\right)^{3} E\left(z(t) \mathbf{x}^{*}(t)\right) \text {. }
\end{aligned}
$$

\section{ACKNOWLEDGMENT}

The authors would like to give thanks to Dr. B. Komiyama, ATR Adaptive Communications Research Laboratories, for his encouragement and helpful discussions. 


\section{REFERENCES}

[1] B. Widrow, P. E. Mantey, L. J. Griffiths, and B. B. Goode, "Adaptive antenna systems," Proc. IEEE, vol. 55, pp. 2143-2159, Dec. 1967.

[2] R. A. Monzingo and T. W. Miller, Introduction to Adaptive Arrays. New York: Wiley, 1980.

[3] J. E. Hudson, Adaptive Array Principles. London, U.K.: Peter Peregrinus, 1981.

[4] Blind Deconvolution, S. Haykin, Ed., PTR Prentice-Hall, Eaglewood Cliffs, NJ, 1994

[5] O. Shalvi and E. Weinstein, "New criteria for blind deconvolution of nonminimum phase systems (channels)," IEEE Trans. Inform. Theory, vol. 36, pp. 312-321, Mar. 1990.

[6] - "Super-exponential methods for blind deconvolution," IEEE Trans. Inform. Theory, vol. 39, pp. 504-519, Mar. 1993.

[7] J. M. Mendel, "Tutorial on higher-order statistics (spectra) in signal processing and system theory: Theoretical results and some applications," Proc. IEEE, vol. 79, pp. 278-305, Mar. 1991.

[8] J. K. Tugnait, "Identification and deconvolution of multichannel linear non-Gaussian processes using higher-order statistics and inverse filter criteria," IEEE Trans. Signal Processing, vol. 45, pp. 658-672, Mar. 1997.

[9] C.-Y. Chi and M.-C. Wu, "Inverse filter criteria for blind deconvolution and equalization using two cumulants," Signal Processing, vol. 43, pp. 55-63, Apr. 1995.

[10] C.-Y. Chi and C.-H. Chen, "Cumulant-based inverse filter criteria for MIMO blind deconvolution: Properties, algorithms, and application to DS/CDMA systems in multipath," IEEE Trans. Signal Processing, vol. 49, pp. 1282-1299, July 2001.

[11] Z. Ding and T. Nguyen, "Stationary points of a Kurtosis maximization algorithm for blind signal separation and antenna beamforming," IEEE Trans. Signal Processing, vol. 48, pp. 1587-1596, June 2000.

[12] M. C. Dogan and J. M. Mendel, "Cumulant-based blind optimum beamforming," IEEE Trans. Aerosp. Electron. Syst., vol. 30, pp. 722-741, July 1994.

[13] E. Gonen and J. M. Mendel, "Applications of cumulants to array processing-Part III: Blind beamforming for coherent signals," IEEE Trans. Signal Processing, vol. 45, pp. 2252-2264, Sept. 1997.

[14] Q. Wu and M. Wong, "Blind adaptive beamforming for cyclostationary signals," IEEE Trans. Signal Processing, vol. 44, pp. 2757-2767, Nov. 1996.

[15] D. N. Godard, "Self-recovering equalization and carrier tracking in two dimensional data communication systems," IEEE Trans. Commun., vol. COM-28, pp. 1867-1875, Nov. 1980

[16] J. R. Treichler and B. G. Agee, "A new approach to multipath correction of constant modulus signals," IEEE Trans. Acoust., Speech, Signal Processing, vol. ASSP-31, pp. 459-472, Apr. 1983.

[17] J. J. Shynk and R. P. Gooch, "The constant modulus array for cochannel signal copy and direction finding," IEEE Trans. Signal Processing, vol. 44, pp. 652-660, Mar. 1996.

[18] J. J. Shynk, A. V. Keerthi, and A. Mathur, "Steady-state analysis of the multistage constant modulus array," IEEE Trans. Signal Processing, vol. 44, pp. 948-962, Apr. 1996.

[19] H. H. Zeng, L. Tong, and C. R. Johnson, "Relationships between the constant modulus and Wiener receivers," IEEE Trans. Inform. Theory, vol. 44, pp. 1523-1538, July 1998 .

[20] B. D. Van Veen and K. M. Buckley, "Beamforming: A versatile approach to spatial filtering," IEEE Acoust., Speech, Signal Processing Mag., pp. 4-24, Apr. 1988.

[21] K. Yang, Y. Zhang, and Y. Mizuguchi, "A signal subspace-based subband approach to space-time adaptive processing for mobile communications," IEEE Trans. Signal Processing, vol. 49, pp. 401-413, Feb. 2001.

[22] K. L. Yeung and S. F. Yao, "A cumulant-based super-exponential algorithm for blind deconvolution of multi-input multi-output systems," Signal Process., vol. 67, no. 2, pp. 141-162, Jan. 1998.

[23] Y. Inouye and K. Tanebe, "Super-exponential algorithms for multichannel blind deconvolution," IEEE Trans. Signal Processing, vol. 48, pp. 881-888, Mar. 2000.

[24] M. Martone, "An adaptive algorithm for antenna array low-rank processing in cellular TDMA base stations," IEEE Trans. Commun., vol. 46, pp. 627-643, May 1998.

[25] G. H. Golub and C. F. Van Loan, Matrix Computations, 3rd ed. Baltimore, MD: Johns Hopkins Univ. Press, 1996.

[26] H. L. Van Trees, Optimum Array Processing. New York: Wiley, 2002, Detection, Estimation, and Modulation Theory.

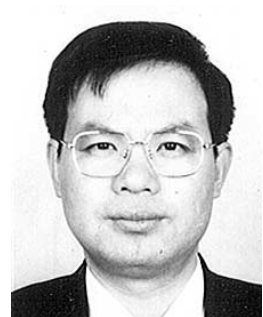

Kehu Yang (M'99) received the B.E., M.S., and $\mathrm{Ph} . \mathrm{D}$. degrees from Xidian University (formerly the Northwest Telecommunications Engineering Institute), Xi'an, China, in 1982, 1984, and 1995, respectively.

He joined Xidian University in 1985, where he became an Associate Professor in May 1996. From December 1998 to May 2002, he was a visiting researcher at ATR Adaptive Communications Research Laboratories, Kyoto, Japan. From June 2002 to October 2002, he was a research fellow at Xi'an Research Institute of ZTE Corporation. From November 2002 to December 2002, he was with Xi' an Haitian Antenna Technologies Co., Ltd., as the leader of the R\&D Department 3. In January 2003, he re-joined Xidian University as an Associate Professor. His main research interests are array signal processing for radar and smart antenna for mobile communications.

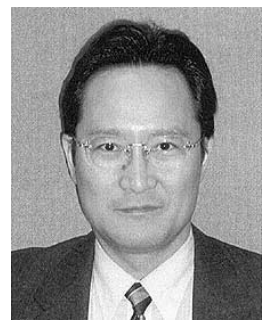

Takashi Ohira (S'79-M'83-SM'99-F'04) was born in Osaka, Japan, in April 1955. He received B.E. and D.E. degrees in communication engineering from Osaka University, in 1978 and 1983, respectively.

In 1983, he joined NTT Electrical Communication Laboratories, Yokosuka, Japan, where he was engaged in research on monolithic integration of microwave semiconductor devices and circuits. He developed GaAs MMIC transponder modules and microwave beamforming networks aboard the Japanese domestic multibeam communication satellites Engineering Test Satellite VI (ETS-VI) and ETS-VIII at NTT Wireless Systems Laboratories, Yokosuka. Since 1999, he has been engaged in research on wireless ad hoc networks and microwave analog adaptive antennas aboard consumer electronic devices at ATR Adaptive Communications Research Laboratories, Kyoto, Japan. Concurrently, he was a Consulting Engineer for the National Astronautical Space Development Agency (NASDA) ETS-VIII Project in 1999 and was an Invited Lecturer at Osaka University from 2000 to 2001. He co-authored Monolithic Microwave Integrated Circuits (Tokyo, Japan: IEICE, 1997)

Dr. Ohira serves as IEEE MTT-S Japan Chapter Vice-Chairperson. He received the 1986 IEICE Shinohara Prize and the 1998 Japan Microwave Prize.

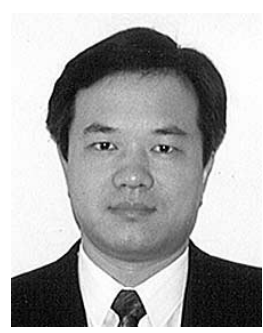

Yimin Zhang (SM'01) received the M.S. and Ph.D. degrees from the University of Tsukuba, Tsukuba, Japan, in 1985 and 1988, respectively.

He joined the faculty of the Department of Radio Engineering, Southeast University, Nanjing, China, in 1988. He served as a Technical Manager at the Communication Laboratory Japan, Kawasaki, Japan, from 1995 to 1997 and was a Visiting Researcher at ATR Adaptive Communications Research Laboratories, Kyoto, Japan, from 1997 to 1998. Since 1998, he has been with the Villanova University, Villanova, PA, where he is currently a Research Associate Professor with the Center for Advanced Communications. His research interests are in the areas of array signal processing, space-time adaptive processing, multiuser detection, blind signal processing, digital mobile communications, and time-frequency analysis. 


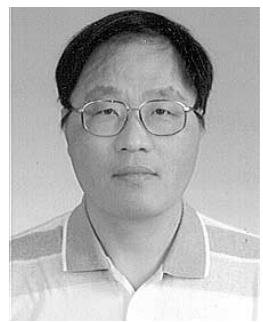

Chong-Yung Chi (S'83-M'83-SM'89) was born in Taiwan, R.O.C., on August 7, 1952. He received the B.S. degree from the Tatung Institute of Technology, Taipei, Taiwan, in 1975, the M.S. degree from the National Taiwan University, Taipei, in 1977, and the $\mathrm{Ph} . \mathrm{D}$. degree from the University of Southern California, Los Angeles, in 1983, all in electrical engineering.

From July 1983 to September 1988, he was with the Jet Propulsion Laboratory, Pasadena, CA, where he worked on the design of various spaceborne radar remote sensing systems including radar scatterometers, synthetic aperture radars, altimeters, and rain mapping radars. From October 1988 to July 1989 , he was a visiting specialist at the Department of Electrical Engineering, National Taiwan University. He has been a Professor with the Departmen of Electrical Engineering since August 1989 and Chairman of Institute of Communications Engineering since August 2002, National Tsing Hua University, Hsinchu, Taiwan. He was a visiting researcher with the Advanced Telecommunications Research (ATR) Institute International, Kyoto, Japan, in May and June 2001. He has published more than 110 technical papers in radar remote sensing, system identification and estimation theory, deconvolution and channel equalization, digital filter design, spectral estimation, and higher order statistics (HOS)-based signal processing. His research interests include signal processing for wireless communications, statistical signal processing, and digital signal processing and their applications.

Dr. Chi is an active member of Society of Exploration Geophysicists, a member of European Association for Signal Processing, and an active member of the Chinese Institute of Electrical Engineering. He was a technical committee member of both the 1997 and 1999 IEEE Signal Processing Workshops on Higher Order Statistics (HOS) and the 2001 IEEE Workshop on Statistical Signal Processing (SSP). He was also a member of International Advisory Committee of TENCON 2001 and a member of International Program Committee of the Fourth International Symposium on Independent Component Analysis and Blind Source Separation (ICA-2003). He was a co-organizer and a general co-chairman of 2001 IEEE SP Workshop on Signal Processing Advances in Wireless Communications (SPAWC-2001), the Internationa Liaison of SPAWC-2003, and Technical Committee Member and International Liaison (Asia and Australia) of SPAWC-2004. He was a Program Committee Member for the International Conference on Signal Processing (ICSP-2003) and a Technical Committee Member of the Third IEEE International Symposium on Signal Processing and Information Technology (ISSPIT'03). He was the Chair of the Information Theory Chapter of the IEEE Taipei Section from July 2001 to June 2003. Currently, he is also an Associate Editor for the IEEE TRANSACTIONS ON Signal PROCESSING and an Editorial Board Member of the EURASIP Journal on Applied Signal Processing. 